\title{
Practical Stabilization of Driftless Systems on Lie Groups: The Transverse Function Approach
}

\author{
Pascal Morin and Claude Samson
}

\begin{abstract}
A general control design approach for the stabilization of controllable driftless nonlinear systems on finite dimensional Lie groups is presented. The approach is based on the concept of bounded transverse functions, the existence of which is equivalent to the system's controllability. Its outcome is the practical stabilization of any trajectory, i.e., not necessarily a solution of the control system, in the state-space. The possibility of applying the approach to an arbitrary controllable smooth driftless system follows in turn from the fact that any controllable homogeneous approximation of this system can be lifted (via a dynamic extension) to a system on a Lie group. Illustrative examples are given.
\end{abstract}

Index Terms-Feedback law, Lie groups, nonlinear systems, stabilization.

\section{INTRODUCTION}

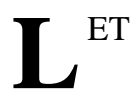

$$
\dot{g}=\sum_{i=1}^{m} u_{i} X_{i}(g)+P(g, t)
$$

denote a control system on a finite-dimensional connected Lie group $G$, with $X_{1}, \ldots, X_{m}$ left-invariant smooth vector fields (v.f.) which satisfy the Lie algebra rank condition (LARC) so that the system is controllable when $P \equiv 0$. The drift term $P(g, t)$ is viewed here as a known or measured perturbation belonging to $G_{g}$ - the tangent space of $G$ at $g$. We assume only the continuity of the function $P$. Obviously, the main case of interest is when $\operatorname{span}\left\{X_{1}(e), \ldots, X_{m}(e)\right\} \neq G_{e}$-when $m<$ $\operatorname{dim}(G)$, for instance. The problem addressed in this paper is the practical stabilization of the identity element $e \in G$ via the asymptotic stabilization of a compact set contained in an arbitrary small neighborhood of $e$.

Prior to commenting upon this particular control objective, it may be useful to explain why the Lie group framework is chosen here. A common motivation is that various physical systems are naturally modeled as systems invariant on Lie groups. Rigid bodies in space and cart-like vehicles are well-known examples. Invariance on a Lie group is a strong geometrical and structural property which is understandably useful for control design purposes. A second motivation is related to the possibility of locally approximating any smooth controllable driftless system

Manuscript received July 15, 2002; revised February 18, 2003. Recommended by Associate Editor W. Kang.

The authors are with the Institut National de Recherche en Automatique et Informatique (INRIA), 06902 Sophia-Antipolis Cedex, France (e-mail:

Pascal.Morin@inria.fr; Claude.Samson@inria.fr).

Digital Object Identifier 10.1109/TAC.2003.816963 by a controllable homogeneous driftless system which can be lifted, via a suitable dynamic extension, to a system invariant on a Lie group. This will be explained in more details further in the paper. After having recalled the generality of systems on Lie groups, our next and final argument is that the Lie group framework is particularly well adapted to the development and exposition of the transverse function (t.f.) control approach presented here. The extensive use of the specific properties associated with systems on Lie groups in the proofs of the main results reported in the present paper is, by itself, a good illustration of this.

Let us now focus on control issues and put the practical stabilization objective in perspective with the research effort devoted to driftless controllable systems. While controllability properties of these systems have been known for a long time-as a consequence of the classical Chow theorem [9]—algorithms to compute open-loop controls in order to steer the system from one point to another have been proposed more recently with various approaches: use of highly oscillatory control inputs [23], [35], [44], explicit calculation of steering trajectories in the case of nilpotent systems [20] and differentially flat systems [13], [27], particularization of these methods to systems on Lie groups [21], possibly with a drift v.f. [6]. This paper does not focus on issues related to open-loop control techniques, such as path planning, or the characterization/construction of optimal paths between two points, or the determination of algorithms which compute "feasible" approximations of a finite-length arbitrary curve. Although the proposed control approach may be useful to address some of these problems in a novel way (the path approximation problem, to cite one of them), its primary application is feedback stabilization. The problem of asymptotic stabilization - via state feedback control- of an equilibrium point of (1), when $P \equiv 0$, has also attracted much attention during the last decade. Many of the studies on the subject have found a challenge and a motivation in Brockett's theorem [5] according to which, if $m<\operatorname{dim}(G)$ and the control v.f. evaluated at $e$ are linearly independent, no smooth or even continuous pure state feedback can make this equilibrium point asymptotically stable. Different types of feedback laws have been considered to circumvent the difficulty—although not all of them guarantee Lyapounov stability. Discontinuous feedbacks [1], [3], [8], [22] and hybrid feedbacks [2], [30], [41] are two possibilities. Another one, more related to the present approach, consists of using continuous time-varying feedbacks [39], [10], [36], [45], [40], [28], [37], [31], [29]. An early survey on the control of nonholonomic systems, whose kinematic models are nonlinear driftless systems, can also be found in [4]. 
The abundance of literature on the subject may convey the feeling that the problems are, by now, well understood and have received satisfactory solutions. We believe on the contrary that some important issues have been left in the shade. One of these issues concerns the compromise between speed of convergence and robustness of the stability property against modeling errors. For a few driftless systems, this type of robustness can be ensured by using a Lipschitz continuous time-varying feedback law [26], but this implies slow—not exponential—convergence to the origin for most of the system's trajectories. On the other hand, to our knowledge and understanding, no robust exponential stabilizer has been proposed until now. It is in fact possible that such a feedback does not exist for systems which do not satisfy Brockett's condition. A result in this direction has been proved in [25]. Efforts to circumvent the difficulty, by considering hybrid continuous/discrete time feedback laws [2], [30], have only brought partial results. For instance, such feedbacks can be made robust to unmodeled dynamics, but stability of the desired equilibrium is not robust against discretization uncertainties. Another issue is related to the trajectory stabilization problem. This problem is usually easier than point stabilization-in particular, the linearized approximation of the associated error system may be controllable - and various feedback solutions have been proposed for specific classes of nonlinear driftless systems, especially in the robotics literature [17], [40], [7]. Asymptotic stabilization is usually obtained under some conditions upon the reference trajectory. Typically, it should not converge to a fixed point. One could have hoped for the existence of a feedback law which would have uniformly guaranteed asymptotic stability independently of the considered trajectory-just as for linear controllable systems-but negative results concerning this existence issue have been proven [24]. This has clear consequences in mobile robotics, when the control objective is the tracking of a reference vehicle whose trajectory is not known in advance.

The aforementionned difficulties suggest to us that, for nonlinear driftless systems, the classical objective of asymptotic stabilization, which underlies a large part of feedback stabilization theory, is at the same time too restrictive and too constraining. The guideline followed in this paper is that practical stabilization can be a more realistic control objective to pursue, and also a touchstone principle for the definition of new approaches for the stabilization of driftless systems. Being less constraining than the one of asymptotic stabilization, it encompasses many control solutions previously proposed in the literature and leaves the door open to other solutions.

The approach here considered for practical stabilization is based on the existence of bounded functions which are transverse to a set of v.f. [34]. Intuitively, one can make a comparison between this approach and the general open-loop control design algorithm developed in [23], [44]. In those papers, the idea was to add virtual control inputs involving sinusoids with fixed and high enough frequencies in order to ensure uniform boundedness of tracking errors by a prespecified threshold. Here, the threshold is directly related to the size of periodic transverse functions whose associated frequencies are the new inputs. In comparison with the approach developed in [23] and [44], we would like to stress that: 1) our approach yields feedback laws;
2) ultimate boundedness of the tracking errors by a prespecified threshold, uniformly w.r.t. the reference trajectory, is guaranteed; and 3) the control frequencies may tend to zero, even when the reference trajectory is not a solution of the control system, so that oscillations are not systematic - this will be illustrated by simulations results at the end of this paper. Let us also mention that the idea of frequency adaptation which underlies the feedback control solutions proposed here can be traced back, in the context of mobile robots, to [12], a work itself adapted from control techniques used for induction motors [11]. Concerning the concept of transversality used throughout this paper, the basic result of equivalence between the existence of a bounded function whose partial derivatives are transversal to a set of smooth v.f. and the satisfaction by these v.f. of the LARC was first proved, to our knowledge, in [34]. However, the way of using this result for control purposes is little developed in that reference, and the importance of the Lie group framework in order to properly develop the t.f. control approach was not identified at that time. For the sake of clarity and precision, a discussion about the contribution of the present paper with respect to [34] is postponed to Remark 5 in Section VI, after the exposition of the main results.

The paper is organized as follows. The t.f. control approach is first illustrated on a simple example in Section II. In the next section, notations used thereafter are specified and a few definitions about invariant systems on Lie groups are recalled. The main technical result about the existence and construction of transverse functions is stated in Theorem 1 in Section IV. Then, in Section V, the concept of transverse function is used to solve the practical stabilization problem evoked at the beginning of this introduction. In Section VI, we show how the approach applies to systems with homogeneous v.f., after precising in a proposition how any homogeneous system can be lifted to a system invariant on a Lie group. Theorem 1 combined with this proposition contains the main result in [34] according to which controllability is equivalent to the existence of transverse functions. Finally, the approach is illustrated by examples in Section VII.

\section{SIMPLE EXAMPLE}

Consider the perturbed three-dimensional chained system with two inputs and state vector $g=\left(g_{1}, g_{2}, g_{3}\right)^{T} \in \mathbb{R}^{3}$

$$
\begin{aligned}
\dot{g} & =u_{1} X_{1}(g)+u_{2} X_{2}+P(g, t) \\
X_{1}(g) & =\left(1,0, g_{2}\right)^{T}, \quad X_{2}=(0,1,0)^{T} .
\end{aligned}
$$

It is known, and simple to verify, that the unperturbed chained system (with $P \equiv 0$ ) is controllable. Consider the following function $f$ which associates an element $\theta$ of the torus $\mathbb{} \mathbb{E} \mathbb{R} / \pi \mathbb{Z}$ with a point in $\mathbb{R}^{3}$

$$
f(\theta)=\left(\varepsilon \sin \theta, \varepsilon \cos \theta, \frac{\varepsilon^{2}}{4} \sin 2 \theta\right)^{T}, \quad \varepsilon>0 .
$$

The Euclidean norm of $f(\theta)$ is clearly uniformly bounded by a number commensurable with $\varepsilon$, and it uniformly tends to zero when $\varepsilon$ tends to zero. This function is of particular interest to us because $\operatorname{det} H(\theta)=-\varepsilon^{2} / 2(\forall \theta)$ with 
$H(\theta) \triangleq\left(X_{1}(f(\theta)) \quad X_{2}(\partial f / \partial \theta)(\theta)\right)$, so that, whenever $\varepsilon \neq 0$, the vectors $X_{1}(f(\theta)), X_{2}$, and $(\partial f / \partial \theta)(\theta)$ form a basis of $\mathbb{R}^{3}$, for every $\theta \in \mathbb{T}$. In other words, the gradient of $f(\theta)$ is transversal to the directions given by $X_{1}$ et $X_{2}$ when these v.f. are evaluated at $g=f(\theta)$. Note that what is remarkable is not so much the property of transversality by itself-the unbounded function $t \in \mathbb{R} \mapsto(0,0, t)^{T} \in \mathbb{R}^{3}$ also trivially satisfies this property-but the combination of transversality and boundedeness of the function $f$. In order to explain the usefulness of such a function for the control of (2), we introduce the following vector:

$$
z \triangleq\left(\begin{array}{c}
g_{1}-f_{1}(\theta) \\
g_{2}-f_{2}(\theta) \\
g_{3}-f_{3}(\theta)-f_{1}(\theta)\left(g_{2}-f_{2}(\theta)\right)
\end{array}\right) .
$$

One easily verifies that, along the solutions of (2)

$$
\dot{z}=C(z, f(\theta))(H(\theta) \bar{u}+D(z) P(g, t))
$$

with

$$
C(z, f)=\left(\begin{array}{ccc}
1 & 0 & 0 \\
0 & 1 & 0 \\
z_{2} & -f_{1} & 1
\end{array}\right) \quad D(z)=\left(\begin{array}{ccc}
1 & 0 & 0 \\
0 & 1 & 0 \\
-z_{2} & 0 & 1
\end{array}\right)
$$

and $\bar{u}=\left(u_{1}, u_{2},-\dot{\theta}\right)^{T}$. Using the fact that both matrices $C(z, f(\theta))$ and $H(\theta)$ are invertible for every $z \in \mathbb{R}^{3}$ and $\theta \in \mathbb{T}$, and viewing $\dot{\theta}$ as an extra control input variable, the preliminary feedback

$$
\bar{u}=H(\theta)^{-1}\left(C(z, f(\theta))^{-1} v-D(z) P(g, t)\right)
$$

with $v$ denoting a free vector in $\mathbb{R}^{3}$, transforms (5) into the simple decoupled linear system

$$
\dot{z}=v
$$

Asymptotic stabilization of the origin of the previous system obviously poses no difficulty. One can take, for example, $v=$ $-k z$ with $k>0$ to ensure exponential stabilization of $z=0$. In view of (4), this control yields exponential convergence of $|g-f(\theta)|$ to zero and, furthermore, asymptotic stabilization of $f(\mathbb{T})$ for (2) if, for insatnce, $\theta(0)=\arg \min _{\theta}|g(0)-f(\theta)|$. It is in this sense that the practical stabilization of $g=0$ is achieved for this system.

Since $z$ is "almost" equal to $g$ when $\varepsilon$ is small, a possible interpretation of the above control solution is that it "nearly" globally linearizes the initial nonlinear system with two control inputs into the trivial linear system $\dot{g}=v$ (quoted words are important here). Following this interpretation, the time derivative of $\theta$ provides the extra control input which allows instantaneous steering in any direction of the state-space. Since $\theta$ is an element of the torus, its time derivative may also be viewed as a frequency variable.

At this point, the specific expression (4) of $z$, the dynamics of which can be globally linearized and decoupled - contrary to the apparently simpler choice $z \triangleq g-f(\theta)$-, may seem a little mysterious. In this respect, the generalization of the approach will reveal the central role played by the Lie group invariance property of the chained system. For instance, we will see that (4) may equally be written as $z \triangleq g \star f(\theta)^{-1}$, with $\star$ denoting the group operation on $\mathbb{R}^{3}$ with respect to which the chained system is invariant, and $f(\theta)^{-1}$ the inverse of $f(\theta)$ with respect to this operation.

\section{RECALLS AND NOTATION}

Let us introduce some notation used hereafter.

- $|\cdot|$ is the Euclidean norm in $\mathbb{R}^{n}$.

- Let $v$ denote a function of the variables $x$ and $y$, we write $v=\mathrm{o}\left(|x|^{k}\right)$ (resp., $\left.v=\mathrm{O}\left(|x|^{k}\right)\right)$ if $\left(v(x, y) /|x|^{k}\right) \rightarrow$ 0 as $|x| \rightarrow 0$ (resp. if $\left(v(x, y) /|x|^{k}\right) \leq K<\infty$ in some neighborhood of $x=0$ ) uniformly with respect to $y$ belonging to compact sets.

- The tangent space of a manifold $M$ at a point $p$ is denoted as $M_{p}$.

- The Lie algebra generated by v.f. $X_{1}, \ldots, X_{m}$ is denoted as $\operatorname{Lie}\left(X_{1}, \ldots, X_{m}\right)$.

- The differential of a smooth mapping $f$ at a point $p$ is denoted as $d f(p)$.

- $\mathbb{T}^{k}$ is the torus of dimension $k$, with $\mathbb{T}=\mathbb{R} / 2 \pi \mathbb{Z}$.

We shall also use standard notation relative to Lie groups; see, e.g., [15] for more details on this topic. Recall that a Lie group is a differentiable manifold endowed with a smooth group operation.

- $G$ denotes a finite-dimensional connected Lie group, with Lie algebra-of left-invariant v.f.- $\mathfrak{g}$. As usual, for the sake of lightening the notation, and unless specifically indicated otherwise, the group product of two elements $\sigma$ and $\tau$ of $G$ will be denoted by $\sigma \tau$.

- The identity element of $G$ is denoted by $e$, i.e., $\forall \tau \in$ $G: \tau e=e \tau=\tau$.

- The inverse of $\tau \in G$ is denoted by $\tau^{-1}$, i.e., $\tau \tau^{-1}=$ $\tau^{-1} \tau=e$.

- Left and right translations are denoted by $l$ and $r$ respectively, i.e., $l_{\sigma}(\tau)=r_{\tau}(\sigma)=\sigma \tau$.

- If $X \in \mathfrak{g}, \exp t X$ is the solution at time $t$ of $\dot{g}=X(g)$ with initial condition $g(0)=e$.

- The adjoint representation of $G$ is Ad, i.e., for $\sigma \in G, \operatorname{Ad}(\sigma)=d I_{\sigma}(e)$ with $I_{\sigma}: G \rightarrow G$ defined by $I_{\sigma}(g)=\sigma g \sigma^{-1}$. By extension, we define $\operatorname{Ad}(\sigma) X(g) \triangleq d l_{g}(e) \operatorname{Ad}(\sigma) X(e)$.

- The differential of Ad is ad, and $(\operatorname{ad} X, Y)=[X, Y]$, the Lie bracket of $X$ and $Y$.

Recall that

- a v.f. $X$ on a Lie group $G$ is left-invariant iff $\forall(\sigma, \tau) \in$ $G^{2}: d l_{\sigma}(\tau) X(\tau)=X(\sigma \tau)$

- a control system $\dot{g}=\sum_{i=1}^{m} u_{i} X_{i}(g)$ on $G$ is said to be left-invariant if the control v.f.'s $X_{i}(1 \leq i \leq m)$ are left-invariant;

- if $g_{1}(\cdot)$ and $g_{2}(\cdot)$ are two solutions of a left-invariant system obtained by applying the same control $u(t)(t \geq 0)$, then $\forall t \geq 0: g_{2}(t)=\tau g_{1}(t)$ with $\tau=g_{2}(0) g_{1}(0)^{-1}$.

For a proper general exposition of the t.f. control approach, the following definition of a graded basis of $\mathfrak{g}$ is also needed.

Definition 1: Let $X_{1}, \ldots, X_{m} \in \mathfrak{g}$ denote independent v.f. such that $\operatorname{Lie}\left(X_{1}, \ldots, X_{m}\right)=\mathfrak{g}$. Define inductively 
$\mathfrak{u}^{k}=\mathfrak{u}^{k-1}+\left[\mathfrak{u}, \mathfrak{u}^{k-1}\right]$ with $\mathfrak{u}=\operatorname{span}\left(X_{1}, \ldots, X_{m}\right)$, and let $K=\min \left\{k: \mathfrak{u}^{k}=\mathfrak{g}\right\}$. A graded basis of $\mathfrak{g}$ associated with $X_{1}, \ldots, X_{m}$ is an ordered basis $\left\{X_{1}, \ldots, X_{n}\right\}$ of $\mathfrak{g}$ associated with two mappings $\lambda, \rho:\{m+1, \ldots, n\} \rightarrow\{1, \ldots, n\}$ such that the following hold.

1) For any $k \in\{1, \ldots, K\}, \mathfrak{u}^{k}=\operatorname{span}\left\{X_{1}, X_{2}, \ldots\right.$, $\left.X_{\operatorname{dim} u^{k}}\right\}$.

2) For $k \geq 2$ and $\operatorname{dim} \mathfrak{u}^{k-1}<i \leq \operatorname{dim} \mathfrak{u}^{k}, X_{i}=$ $\left[X_{\lambda(i)}, X_{\rho(i)}\right]$ with $X_{\lambda(i)} \in \mathfrak{u}^{a}, X_{\rho(i)} \in \mathfrak{u}^{b}$, and $a+b=k$.

With any graded basis of $\mathfrak{g}$, one can associate a weight vector $\left(r_{1}, \ldots, r_{n}\right)$ defined by

$$
r_{i}=k \Leftrightarrow X_{i} \in \mathfrak{u}^{k} \backslash \mathfrak{u}^{k-1} \Leftrightarrow \operatorname{dim} \mathfrak{u}^{k-1}+1 \leq i \leq \operatorname{dim} \mathfrak{u}^{k}
$$

It follows from Definition 1 that $1=r_{1}=\cdots=r_{m}<r_{m+1} \leq$ $\cdots \leq r_{n}=K$ and, $\forall i>m, r_{i}=r_{\lambda(i)}+r_{\rho(i)}$.

\section{TRANSVERSE FUNCTIONS}

The main result about the existence and construction of transverse functions is the following.

Theorem 1: Let $G$ denote a Lie group of dimension $n$ with Lie algebra $\mathfrak{g}$. Let $X_{1}, \ldots, X_{m} \in \mathfrak{g}$ denote independent v.f. Then, the following properties are equivalent.

1) $\operatorname{Lie}\left(X_{1}, \ldots, X_{m}\right)=\mathfrak{g}$.

2) For any neighborhood $\mathcal{U}$ of $e$ in $G$, there exists a function $f \in \mathcal{C}^{\infty}\left(\mathbb{T}^{n-m} ; \mathcal{U}\right)$ such that, for any $\theta \in \mathbb{T}^{n-m}$

$G_{f(\theta)}=\operatorname{span}\left\{X_{1}(f(\theta)), \ldots, X_{m}(f(\theta))\right\} \oplus d f(\theta)\left(\mathbb{T}_{\theta}^{n-m}\right)$.

Furthermore, with $\left\{X_{1}, \ldots, X_{n}\right\}$ denoting a graded basis of $\mathfrak{g}$, a possible choice for $f$ is given by

$$
\begin{aligned}
\forall \theta & =\left(\theta_{m+1}, \ldots, \theta_{n}\right) \in \mathbb{T}^{n-m} \\
f(\theta) & =f_{n}\left(\theta_{n}\right) f_{n-1}\left(\theta_{n-1}\right) \ldots f_{m+1}\left(\theta_{m+1}\right)
\end{aligned}
$$

with $f_{j}: \mathbb{T} \rightarrow G$ defined by

$$
f_{j}\left(\theta_{j}\right)=\exp \left(\varepsilon_{j}^{r_{\lambda(j)}} \sin \theta_{j} X_{\lambda(j)}+\varepsilon_{j}^{r_{\rho(j)}} \cos \theta_{j} X_{\rho(j)}\right)
$$

for some positive real numbers $\varepsilon_{m+1}, \ldots, \varepsilon_{n}$.

As in [34], functions which satisfy (8) are called "transverse" to the v.f. $X_{1}, \ldots, X_{m}$, or just transverse functions when no ambiguity is possible.

Theorem 1 is coordinate-free. When providing $G$ with a system of coordinates in $\mathbb{R}^{n}$, relation (8) means that the square matrix

$$
H(\theta)=\left(X_{1}(f(\theta)) \ldots X_{m}(f(\theta)) \frac{\partial f}{\partial \theta_{m+1}}(\theta) \cdots \frac{\partial f}{\partial \theta_{n}}(\theta)\right)
$$

is invertible for every $\theta$.

There are many ways to derive transverse functions; see, for instance, [33] for an alternative expression of such a function. Also, in (10), one can introduce two parameters $\varepsilon_{j, 1}$ and $\varepsilon_{j, 2}$, instead of the single parameter $\varepsilon_{j}$. This yields

$$
f_{j}\left(\theta_{j}\right)=\exp \left(\varepsilon_{j, 1}^{r_{\lambda(j)}} \sin \theta_{j} X_{\lambda(j)}+\varepsilon_{j, 2}^{r_{\rho(j)}} \cos \theta_{j} X_{\rho(j)}\right)
$$

The authors know from their experience in robotics that this extra degree of freedom is useful in practice. Another possibility, also important in practice because it can significantly reduce the calculation complexity, consists in using, in either (10) or (12), v.f. $X_{\lambda(j)}$ and $X_{\rho(j)}$ associated with a suitable homogeneous approximation of the control system. This is illustrated by the example of a unicycle in Section VII.

The choice of the parameters $\varepsilon_{m+1}, \ldots, \varepsilon_{n}$ is further specified in Lemma 3 used in the proof of the theorem.

Proof of Theorem 1: For the proof of (Property $2 \Rightarrow$ Property 1), we refer the reader to [34]. This is a direct consequence of the Frobenius Theorem. To prove that Property 1 implies Property 2, we only have to show that (8) is satisfied with the function $f$ defined by (9)-(10), for some values of the parameters $\varepsilon_{m+1}, \ldots, \varepsilon_{n}$. We indicate later the main steps of the proof in the form of three lemmas.

By standard calculations, we first prove the following.

Lemma 1: There exist analytic functions $v_{i, j}(i \in$ $\{1, \ldots, n\}, j \in\{m+1, \ldots, n\})$ such that

$$
\frac{\partial f_{j}}{\partial \theta_{j}}\left(\theta_{j}\right)=\sum_{i=1}^{n} v_{i, j}\left(\theta_{j}\right) X_{i}\left(f_{j}\left(\theta_{j}\right)\right)
$$

with

$$
v_{i, j}= \begin{cases}\mathrm{O}\left(\left|\varepsilon_{j}\right|^{r_{i}}\right) & \forall i \\ \mathrm{O}\left(\left|\varepsilon_{j}\right|^{r_{i}}\right) & \text { if } i<j \quad \text { and } r_{i}=r_{j} \\ \frac{\varepsilon_{j}^{r_{j}}}{2}+\mathrm{o}\left(\left|\varepsilon_{j}\right|^{r_{j}}\right) & \text { if } i=j\end{cases}
$$

From this lemma, we then prove the following.

Lemma 2: There exist analytic functions $a_{i, j}(i \in$ $\{1, \ldots, n\}, j \in\{m+1, \ldots, n\})$ such that

$$
\frac{\partial f}{\partial \theta_{j}}(\theta)=\sum_{i=1}^{n} a_{i, j}(\theta) X_{i}(f(\theta))
$$

with

$$
a_{i, j}=\left\{\begin{array}{l}
\mathrm{O}\left(\left|\bar{\varepsilon}_{j}\right|^{r_{i}}\right) \quad \forall i \\
\sum_{k<r_{i}} \mathrm{O}\left(\left|\varepsilon_{j}\right|^{k}\right) \mathrm{O}\left(\left|\bar{\varepsilon}_{j-1}\right|^{r_{i}-k}\right)+\mathrm{O}\left(\left|\bar{\varepsilon}_{j}\right|^{r_{i}}\right) \\
\quad \text { if } i<j \quad \text { and } r_{i}=r_{j} \\
\frac{\varepsilon_{j}^{r_{j}}}{2}+\sum_{k<r_{j}} \mathrm{O}\left(\left|\varepsilon_{j}\right|^{k}\right) \mathrm{O}\left(\left|\bar{\varepsilon}_{j-1}\right|^{r_{j}-k}\right)+\mathrm{o}\left(\left|\bar{\varepsilon}_{j}\right|^{r_{j}}\right) \\
\quad \text { if } i=j
\end{array}\right.
$$

where $\bar{\varepsilon}_{k} \triangleq\left(\varepsilon_{m+1}, \ldots, \varepsilon_{k}\right)$ if $k=m+1, \ldots, n$, and $\bar{\varepsilon}_{m} \triangleq 0$.

Note that, if all $\mathrm{O}$ and o terms in the previous expressions were equal to zero, then Theorem 1 would follow directly from (15)-(16) and from the fact that $\left\{X_{1}, \ldots, X_{n}\right\}$ is a basis of $\mathfrak{g}$. Although this is not the case, it is not very difficult to show that these terms can be neglected provided that the $\varepsilon_{j}$ 's are adequately chosen, as stated in the following lemma.

Lemma 3: There exist numbers $\beta_{m+1}, \ldots, \beta_{n}$, and $\varepsilon_{0}>0$, such that choosing $\left(\varepsilon_{m+1}, \ldots, \varepsilon_{n}\right)=\varepsilon\left(\beta_{m+1}, \ldots, \beta_{n}\right)$ with $0<\varepsilon<\varepsilon_{0}$, yields

$$
\forall \theta \in \mathbb{T}^{n-m} \quad \operatorname{det}\left(a_{i, j}(\theta)\right)_{i, j=m+1, \ldots, n} \neq 0 .
$$

In view of (15), (17) is equivalent to (8), so that the proof of the theorem follows. The proofs of the previous lemmas are reported in Appendix A.

Q.E.D. 
Note that Lemma 3 does not state that "any" set $\left(\varepsilon_{m+1}, \ldots, \varepsilon_{n}\right)$ of small enough numbers is suitable, nor does it imply that these numbers have to be small. For instance, if the v.f. $X_{1}, \ldots, X_{m}$ are homogeneous w.r.t. some dilation, the lemma holds for any $\varepsilon_{0}>0$.

\section{ApPlicAtion to PracticAl StabiLIZATiON}

\section{A. Point Stabilization}

Consider (1) and assume-without loss of generalitythat $X_{1}, \ldots, X_{m}$ are independent. We show in the following proposition how the concept of transverse functions can be used to design control laws for System (1) that make $g=e$ practically stable. Let us remark that no assumption is made on $P$. For instance, when the projection of $P(e, t)$ onto $\left(\operatorname{span}\left\{X_{1}(e), \ldots, X_{m}(e)\right\}\right)^{\perp}$ does not tend to zero as $t$ tends to infinity, no control law can make $g=e$ an equilibrium of the system, and asymptotic stabilization of this element is pointless.

Proposition 1: Let $f: \mathbb{T}^{n-m} \rightarrow \mathcal{U}$ denote a transverse function. Then along any solution $g(\cdot)$ of (1), and along any trajectory $\theta(\cdot)$ in $\mathbb{T}^{n-m}$

$$
\begin{aligned}
\dot{z}=d r_{f(\theta)^{-1}}(g) d l_{z}(f(\theta)) & \left(\sum_{i=1}^{m} u_{i} X_{i}(f(\theta))\right. \\
& \left.-\sum_{j=m+1}^{n} \dot{\theta}_{j} \frac{\partial f}{\partial \theta_{j}}(\theta)+d l_{z^{-1}}(g) P(g, t)\right)
\end{aligned}
$$

with $z \triangleq g f(\theta)^{-1}$. Moreover, if $Z$ is a v.f. on $G$, then the dynamic feedback law $(u, \dot{\theta})(\theta, g, t)$ defined by

$$
\begin{aligned}
\sum_{i=1}^{m} u_{i}(\theta, g, t) X_{i} & (f(\theta))-\sum_{j=m+1}^{n} \dot{\theta}_{j}(\theta, g, t) \frac{\partial f}{\partial \theta_{j}}(\theta) \\
& =d l_{z^{-1}}(g)\left(d r_{f(\theta)}(z) Z(z)-P(g, t)\right)
\end{aligned}
$$

yields the satisfaction of the following equation:

$$
\dot{z}=Z(z)
$$

along any solution of the controlled system.

Proof: The proof of (18) is easily obtained by differentiating the equality $z f=g$ and using the identities $\left(d l_{z}(f)\right)^{-1}=$ $d l_{z^{-1}}(g),\left(d r_{f}(z)\right)^{-1}=d r_{f^{-1}}(g)$. By applying the feedback law (19) to (1), one deduces (20) from (18).

Q.E.D.

An immediate corollary of the aforementioned proposition is that it suffices to choose for $Z$ any v.f. which asymptotically stabilizes $e$ to ensure asymptotic stability of this point for the system (20) and, therefore, convergence of $g$ to $f\left(\mathbb{T}^{n-m}\right)$ for (1). Furthermore, asymptotic stability of $f\left(\mathbb{T}^{n-m}\right)$ for (1) controlled by the feedback defined by (19) is also granted, provided that $\theta(0)=\arg \min _{\theta} d(g(0), f(\theta))$ with $d$ some left-invariant metric on $G$.

Remark 1: Equations (18) and (19) are the coordinate-free generalizations of (5) and (6) derived for the three-dimensional chained system. They hold whatever the system of coordinates chosen to represent the elements of $G$ as vectors in $\mathbb{R}^{n}$.
Remark 2: (Explicit Control Expression): The explicit calculation of the feedback control defined by (19) will often require the preliminary choice of a system of coordinates. Once this choice is made, if one keeps the same letters to denote the v.f. involved in (19) and their (local) representations in $\mathbb{R}^{n}$, then (19) is equivalent to

$$
\left(\begin{array}{c}
u \\
-\dot{\theta}
\end{array}\right)=H(\theta)^{-1} d l_{z^{-1}}(g)\left(d r_{f(\theta)}(z) Z(z)-P(g, t)\right)
$$

with $H(\theta) \in \mathbb{R}^{n \times n}$ the invertible matrix defined by (11), $d l_{z^{-1}}(g)=(\partial \phi / \partial y)\left(z^{-1}, g\right)$, and $d r_{f}(z)=(\partial \phi / \partial x)(z, f)$, with $\phi(x, y)$ denoting the local representation in $\mathbb{R}^{n}$ of the point $x y$. Equation (21) is the coordinate-dependent generalization of (6).

Remark 3: (Output Linearization and Associated Zero Dynamics): Since the choice of $Z$ in the control expression is free, one can interpret this v.f. as a free control vector and (21) as a feedback control which, in view of (20), "almost linearizes" the equations of the original system. A clear advantage of this type of linearization over "exact" feedback linearization is that it is free of singularities. Another one is the decoupled form of the resulting linear system and, as a matter of fact, its extreme simplicity. This interpretation is conceptually attractive, but it is important to realize that it does not "tell" everything about the approach. For instance, it leaves in the shade the dynamics associated with the extra set of variables $\theta$, while these "hidden" dynamics may have their importance in the overall evaluation of the control performance. For example, if the convergence of $z$ to zero results in large sustained values of $\dot{\theta}$ then highly oscillatory motion of $g$ will take place. This may not be desirable for a certain number of applications. On the other hand, oscillatory motion may also correspond to a requirement of the application. Just consider the case of a car having to perform a small lateral motion within a given time period. This can only be done via one or several maneuvers whose number and amplitude are clearly related to the frequency of the steering-wheel angular velocity. Typically, the smaller the amplitude the larger the frequency will need to be.

Remark 4: (About the Convergence of $\dot{\theta}$ to Zero): Proposition 1 indicates how any v.f. $Z$ which asymptotically stabilizes the identity element of $G$ induces a feedback law for System (1) which asymptotically stabilizes the set $f\left(\mathbb{T}^{n-m}\right)$. For instance, given a system of coordinates on $G$, exponential stability is obtained by choosing $Z(z)=K z$, with $K$ any Hurwitz stable matrix. Note that uniform asymptotic boundedness of all control variables is automatically ensured. As already mentioned, a complementary analysis of the zero dynamics associated with $z=0$ is usually necessary to deduce other properties of the closed-loop system. The equations of this zero dynamics are just obtained by setting $z=0$ in the control expression (21). In the specific case when the perturbation $P(g, t)$ is identically equal to zero, or when it does not depend upon $g$ while $P(t)$ tends to zero, these equations simplify to $(u=0, \dot{\theta}=0)$ and one deduces that all control variables converge to zero. This implies in particular, that $(\dot{g}, \dot{\theta})$ tends to zero. Note that this does not necessarily mean that the state itself converges to some point. However, it is not difficult to prove that such is the case when $P$ is identically equal to zero, 
or when $P(t)$ tends exponentially to zero, and $z$ converges exponentially to zero. Now, let us point out that $\dot{\theta}$ may also tend to zero when the perturbation $P$ does not vanish. In view of (19), this requires the existence of elements $\theta \in \mathbb{T}^{n-m}$ such that $P(f(\theta), t) \in$ span $\left\{X_{1}(f(\theta)), \ldots, X_{m}(f(\theta))\right\}(\forall t)$. The possibility of having the extended state $(g, \theta)$ converge when $P$ does not vanish will be illustrated along the unicycle example treated further in this paper.

\section{B. Trajectory Stabilization}

We show how Proposition 1 directly applies to the problem of practical stabilization of a trajectory on a Lie group. Let $g_{r}(\cdot)$ denote an arbitrary smooth trajectory in $G$. For any basis $X_{1}, \ldots, X_{n}$ of $\mathfrak{g}$, there exist smooth functions $v_{r, i}(i=1, \ldots, n)$ such that

$$
\dot{g}_{r}=\sum_{i=1}^{n} v_{r, i} X_{i}\left(g_{r}\right) .
$$

Furthermore, if $Y_{1}, \ldots, Y_{n}, Z_{1}, \ldots, Z_{n}$ are left-invariant v.f. in $\mathfrak{g}$ and $\sigma, \tau$ are solutions to the differential equations

$$
\dot{\sigma}=\sum_{i=1}^{n} v_{i} Y_{i}(\sigma) \quad \dot{\tau}=\sum_{i=1}^{n} u_{i} Z_{i}(\tau)
$$

then the following identity holds:

$$
\frac{d}{d t}\left(\sigma^{-1} \tau\right)=\sum_{i=1}^{n} u_{i} Z_{i}\left(\sigma^{-1} \tau\right)-\sum_{i=1}^{n} v_{i} \operatorname{Ad}\left(\tau^{-1} \sigma\right) Y_{i}\left(\sigma^{-1} \tau\right) .
$$

One deduces the following error system associated with the trajectory stabilization problem:

$$
\begin{aligned}
\dot{\tilde{g}} & =\sum_{i=1}^{m} u_{i} X_{i}(\tilde{g})-\sum_{i=1}^{n} v_{r, i}(t) \operatorname{Ad}\left(\tilde{g}^{-1}\right) X_{i}(\tilde{g}) \\
& =\sum_{i=1}^{m} u_{i} X_{i}(\tilde{g})+P(\tilde{g}, t)
\end{aligned}
$$

with $\tilde{g} \triangleq g_{r}^{-1} g$ representing the tracking error and

$$
P(\tilde{g}, t) \triangleq-\sum_{i=1}^{n} v_{r, i}(t) \operatorname{Ad}\left(\tilde{g}^{-1}\right) X_{i}(\tilde{g}) .
$$

Since this system has the same form as (1), Proposition 1 applies to it and provides control laws which ensure asymptotic stabilization of the set $f\left(\mathbb{T}^{n-m}\right)$ for the error system (22). It is in this sense that practical stabilization of the trajectory $g_{r}(\cdot)$ is achieved. In particular, if $d(\cdot, \cdot)$ denotes a left invariant Riemannian distance on $G$, then the practical stabilization of $g_{r}(\cdot)$ implies that, for any solution of the controlled system, $d\left(g(t), g_{r}(t)\right)-d(f(\theta(t)), e)$ asymptotically tends to zero.

\section{CASE OF Homogeneous Systems}

In this section, we show how the results of the previous sections apply to driftless controllable systems with homogeneous v.f. The study of such systems is motivated by several reasons. One of them is that any controllable smooth driftless control system on $\mathbb{R}^{n}$ can be approximated by a controllable system with homogeneous v.f. [42], [16]. While this approximation is local in general, there are also physical systems which admit an homogeneous representation in a large domain. The modeling by chained systems of the kinematic equations of several nonholonomic wheeled mobile robots is a well-known example.

The main tool used to apply the results of the previous sections to homogeneous systems is the so-called lifting theorem [38] which specifies how homogeneous systems can be viewed as systems on Lie groups. This explains in part the importance given to Lie groups in the formal Lie-algebraic literature [19], [43]. In this literature, free Lie algebras and free systems [18] are usually considered. They correspond to the framework for the original lifting theorem where nilpotent v.f. are lifted to a free Lie group. While this is well justified from a theoretical standpoint and the sake of generality, it can be interesting, for practical purposes and computational efficiency, to lift the v.f. associated with a specific control system under consideration to the smallest possible Lie group, i.e., the embedding Lie group with the smallest dimension. This possibility, investigated in [14], will be used here.

\section{A. Lifting of an Homogeneous System to a System on a Lie Group}

Prior to stating, in the form of a proposition, a version of the lifting theorem adapted to our present objectives, let us recall a few basic definitions and properties about homogeneity (for more details, we refer the reader to [16]).

Given $\lambda>0$ and a weight vector $r=\left(r_{1}, \ldots, r_{n}\right)\left(r_{i}>\right.$ $0 \forall i)$, a dilation $\Delta_{\lambda}^{r}$ on $\mathbb{R}^{n}$ is a map from $\mathbb{R}^{n}$ to $\mathbb{R}^{n}$ defined by $\Delta_{\lambda}^{r} x \triangleq\left(\lambda^{r_{1}} x_{1}, \ldots, \lambda^{r_{n}} x_{n}\right)$. A function $f \in \mathcal{C}^{0}\left(\mathbb{R}^{n} ; \mathbb{R}\right)$ is homogeneous of degree $d$ with respect to the family of dilations $\left(\Delta_{\lambda}^{r}\right)_{\lambda>0}$, or more concisely $\Delta^{r}$-homogeneous of degree $d$, if $\forall \lambda>0, f\left(\Delta_{\lambda}^{r} x\right)=\lambda^{d} f(x)$. A smooth v.f. $X$ on $\mathbb{R}^{n}$ is $\Delta^{r}$ homogeneous of degree $\tau$ if, for any $i=1, \ldots, n$, the function $x \longmapsto X_{i}(x)$ is $\Delta^{r}$-homogeneous of degree $\tau+r_{i}$.

The possibility of lifting a set of homogeneous v.f. to a Lie group, with dimension equal to the dimension of the Lie algebra generated by the v.f., is summarized in the following proposition.

Proposition 2: [38], [14]: Let $X_{1}, \ldots, X_{m}$ denote smooth v.f. on $\mathbb{R}^{n}$, independent ${ }^{1}$ over $\mathbb{R}, \Delta^{r}$-homogeneous of degree $-\tau_{1}, \ldots,-\tau_{m}<0$ respectively, and which satisfy the LARC at the origin. Let $\bar{n}$ denote the dimension, over $\mathbb{R}$, of $\operatorname{Lie}\left(X_{1}, \ldots, X_{m}\right)$. Then, there exist

i) a lifting $\bar{X}_{i}$ of $X_{i}$ on $\mathbb{R}^{\bar{n}}(1 \leq i \leq m)$ of the following form:

$$
\forall \bar{x}=(x, y) \in \mathbb{R}^{\bar{n}} \quad \bar{X}_{i}(\bar{x})=\left(\begin{array}{c}
X_{i}(x) \\
Y_{i}(x, y)
\end{array}\right) ;
$$

ii) a smooth mapping $\varphi: \mathbb{R}^{\bar{n}} \times \mathbb{R}^{\bar{n}} \rightarrow \mathbb{R}^{\bar{n}}$; such that

1) $\bar{X}_{1}, \ldots, \bar{X}_{m}$ satisfy the LARC at the origin;

2) $\bar{X}_{1}, \ldots, \bar{X}_{m}$ are $\Delta^{\bar{r}}$-homogeneous of degree $-\tau_{1}, \ldots$, $-\tau_{m}$, respectively, for some $\bar{r} \in \mathbb{R}^{\bar{n}}$;

${ }^{1}$ That is, $\left(\sum_{i=1}^{m} \alpha_{i} X_{i}=0\right.$ with $\left.\alpha_{i} \in \mathbb{R}, i=1, \ldots, m\right) \Longrightarrow\left(\alpha_{i}=\right.$ $0, \forall i)$. 
3) $\mathbb{R}^{\bar{n}}$, endowed with the composition law $(\bar{x}, \bar{y}) \longmapsto \bar{x} \star \bar{y} \triangleq$ $\varphi(\bar{x}, \bar{y})$ is a Lie group, and $\bar{X}_{1}, \ldots, \bar{X}_{m}$ are left-invariant v.f. for this composition law.

For a constructive proof of this proposition, the interested reader is referred to [33].

Remark 5: The combination of Theorem 1 and Proposition 2 implies the results stated in Theorem 1 of [34] and, in particular, the existence of transverse functions for any smooth controllable driftless system. It also yields complementary results. One of them is the specification of a smaller number of variables on which transverse functions depend. More precisely, this number is equal to the difference between the dimension of the Lie algebra generated by the v.f. of a controllable homogeneous approximation of the system and the number of independent control v.f. Another important complementary result is the expressions (9)-(10) of such a transverse function. This expression is much more concise and explicit than the construction proposed in the proof of Theorem 1 in [34]. Finally, let us mention that no general control expression was given in [34]. In this respect the Lie group structure is essential.

The next section explains how to use Proposition 2 in conjunction with Proposition 1 to achieve practical stabilization of a controllable homogeneous driftless system.

\section{B. Application to Practical Stabilization}

Consider a $n$-dimensional controllable homogeneous driftless system perturbed by an additive v.f. $P(x, t)$

$$
\dot{x}=\sum_{i=1}^{m} u_{i} X_{i}(x)+P(x, t)
$$

The control v.f. $X_{1}, \ldots, X_{m}$ are assumed to be independent over $\mathbb{R}$ so that all assumptions in Proposition 2 are satisfied. Let $\bar{X}_{1}, \ldots, \bar{X}_{m}$ be the v.f. evoked in this proposition. A dynamic extension of system (24) is then the $\bar{n}$-dimensional system

$$
\dot{\bar{x}}=\sum_{i=1}^{m} u_{i} \bar{X}_{i}(\bar{x})+\bar{P}(\bar{x}, t)
$$

with

$$
\begin{aligned}
\bar{x} & =\left(\begin{array}{c}
x \\
y
\end{array}\right) \quad \bar{X}_{i}(\bar{x})=\left(\begin{array}{c}
X_{i}(x) \\
Y_{i}(x, y)
\end{array}\right) \\
\bar{P}(\bar{x}, t) & =\left(\begin{array}{c}
P(x, t) \\
0
\end{array}\right) .
\end{aligned}
$$

According to Proposition 2, the corresponding unperturbed system (obtained by setting $P \equiv 0$ ) is left invariant on the Lie group $\mathbb{R}^{\bar{n}}$ endowed with the group operation $\star$ defined in the proposition. Therefore, Proposition 1 applies directly to system (25), yielding a feedback control $(u(\bar{x}, \theta, t), \dot{\theta}(\bar{x}, \theta, t))$ which ensures (global) practical stabilization of the origin, i.e., the identity element, of $\mathbb{R}^{\bar{n}}$ for this system. The expression of the control law depends on a transverse function $\bar{f}: \mathbb{T}^{\bar{n}-m} \rightarrow \mathbb{R}^{\bar{n}}$ associated with the v.f. $\bar{X}_{1}, \ldots, \bar{X}_{m}$, and Theorem 1 provides an example of such a transverse function. From there, it remains to specify conditions under which the obtained controller is also a (global) practical stabilizer for the initial system (24).
Recall that the origin of $\mathbb{R}^{\bar{n}}$ is practically stabilized in the sense that the set $\bar{f}\left(\mathbb{T}^{\bar{n}-m}\right)$ is asymptotically stable for the extended system (25), after closing the loop with the feedback control and choosing $\theta(0)$ adequately. This implies that the set $\bar{f}_{x}\left(\mathbb{T}^{\bar{n}-m}\right)$-with $\bar{f}_{x}$ denoting the first $n$ components of the vector-valued function $\bar{f}$-is (globally) attractive for the closed-loop solutions of (24). Then, by using classical norm inequalities one easily verifies that, in order to further ensure stability of this set, it suffices to choose the initial condition $y(0)$ equal to $\bar{f}_{y}(\theta(0))$ - with $\bar{f}_{y}$ denoting the remaining $(\bar{n}-n)$ components of the vector-valued function $\bar{f}$.

\section{EXAMPLES}

\section{A. Chained Systems}

The (perturbed) chained system of dimension $n$ with two inputs is defined by

$$
\dot{x}=u_{1} X_{1}(x)+u_{2} X_{2}+P(x, t)
$$

with $X_{1}(x)=\left(1,0, x_{2}, \ldots, x_{n-1}\right)^{T}, X_{2}=(0,1,0, \ldots, 0)^{T}$, and $P$ some additive drift term. The unperturbed chained system-corresponding to the case where $P \equiv 0$ - is a particular homogeneous system, and it is well known that $\operatorname{Lie}\left(X_{1}, X_{2}\right)$ is of dimension $n$, and is spanned by the v.f. $X_{1}, \ldots, X_{n}$ with

$$
X_{i} \triangleq\left(\operatorname{ad}^{i-2} X_{1}, X_{2}\right)=(-1)^{i} e_{i} \quad(i=3, \ldots, n)
$$

and $e_{i}=(0, \ldots, 0,1,0, \ldots, 0)$ the $i$ th canonical vector on $\mathbb{R}^{n}$. According to Proposition 2, $X_{1}$ and $X_{2}$ are left-invariant w.r.t. a group operation on $\mathbb{R}^{n}$. One easily verifies that this group operation is given by

$$
x \star y=\varphi(x, y)=e^{A y_{1}} x+y
$$

with $A$ the $n \times n$ matrix whose only nonzero entries are $a_{i+1, i}=$ 1 , for $i=2, \ldots, n-1$. More explicitly, the components of $x \star y$ are defined by

$$
(x \star y)_{i}= \begin{cases}x_{i}+y_{i}, & \text { if } i=1,2 \\ x_{i}+y_{i}+\sum_{j=2}^{i-1} \frac{y_{1}^{i-j}}{(i-j) !} x_{j}, & \text { otherwise. }\end{cases}
$$

Let us now proceed with the computation of transverse functions for this system. $X_{1}$ and $X_{2}$ complemented with the $n-2$ v.f. defined by (27) form a graded basis of $\operatorname{Lie}\left(X_{1}, X_{2}\right)$ in the sense of Definition 1, and the associated weight-vector is given by $r=(1,1,2, \ldots, n-1)$. Therefore, if we use (10) to calculate each $f_{j}$, we deduce from (27) that

$$
f_{j}\left(\theta_{j}\right)=\exp \left(\varepsilon_{j} \sin \theta_{j} X_{1}+\varepsilon_{j}^{j-2} \cos \theta_{j} X_{j-1}\right) .
$$

From the definition of $X_{1}, X_{2}$, and (27), it is not difficult to obtain

$$
\begin{aligned}
f_{j}\left(\theta_{j}\right)= & (-1)^{j-1}\left((-1)^{j+1} \varepsilon_{j} \sin \theta_{j}, 0, \ldots, 0, \varepsilon_{j}^{j-2} \cos \theta_{j}\right. \\
& \left.\varepsilon_{j}^{j-1} \frac{\sin \theta_{j} \cos \theta_{j}}{2}, \ldots, \varepsilon_{j}^{n-1} \frac{\left(\sin \theta_{j}\right)^{n-j+1} \cos \theta_{j}}{(n-j+2) !}\right)^{T} .
\end{aligned}
$$


The expression of $f=f_{n} \star \cdots \star f_{3}$ can then be calculated from (29). Then, for any transverse function, and any Hurwitz stable matrix $K$, the choice $Z(z)=K z$ in the control expression (21) yields the feedback

$$
\left(\begin{array}{c}
u \\
-\dot{\theta}
\end{array}\right)=H^{-1}(\theta)(w(x, f(\theta), t)
$$

$$
\left.-A(x-f(\theta)) w_{1}(x, f(\theta), t)\right)
$$

with $H(\theta)$ defined, as before, by (11)

$$
w(x, f(\theta), t)=e^{A f_{1}} K e^{-A f_{1}}(x-f(\theta))-P(x, t)
$$

and $w_{1}, f_{1}$, denoting the first component of $w$ and $f$ respectively. This control is a practical stabilizer of (26). This was the main result of [32] —up to a small difference due to inverting the roles played by $z$ and $z^{-1}$, knowing that both choices $z \triangleq g f^{-1}$ and $z \triangleq f g^{-1}$ can be made.

\section{B. Systems on $\mathbf{S O}(\mathbf{3})$ With Two Control Inputs}

Recall that $\mathbf{S O}(\mathbf{3})$ is the set of rotation matrices in $\mathbb{R}^{3}$. Endowed with the classical product of matrices, it is a three-dimensional Lie group. Its Lie algebra consists of the set of $(3 \times 3)$ skew-symmetric real matrices and is classically denoted as $\mathfrak{s o}(3)$. Moreover, the exponential of an element of $\mathfrak{s o}(3)$ coincides with the classical matrix exponential of this element. Consider the following underactuated control system on this group:

$$
\dot{R}=R\left(u_{1} S\left(e_{1}\right)+u_{2} S\left(e_{2}\right)\right)
$$

where $e_{i}$ denotes, as in the above example, the $i$ th canonical vector in $\mathbb{R}^{3}$, and $S$ is the operator associated with the vector product, i.e., $S(e) x=e \times x$. Since $S\left(e_{1}\right), S\left(e_{2}\right)$, and

$$
S\left(e_{3}\right)=\left[S\left(e_{1}\right), S\left(e_{2}\right)\right]=S\left(e_{1}\right) S\left(e_{2}\right)-S\left(e_{2}\right) S\left(e_{1}\right)
$$

form a basis of $\mathfrak{s o}(3)$, the Lie algebra generated by $R S\left(e_{1}\right)$ and $R S\left(e_{2}\right)$ is isomorphic to $\mathfrak{s o}(3)$. This basis is a graded basis in the sense of Definition 1, with $r=(1,1,2)$. We calculate a transverse function by (9)-(10), i.e.,

$$
\begin{aligned}
F(\theta) & =\exp \left(\varepsilon \sin \theta S\left(e_{1}\right)+\varepsilon \cos \theta S\left(e_{2}\right)\right) \\
& =\exp \left(S\left(\varepsilon \sin \theta e_{1}+\varepsilon \cos \theta e_{2}\right)\right) \\
& =\exp \left(S\left(-\theta e_{3}\right)\right) \exp \left(S\left(\varepsilon e_{2}\right)\right) \exp \left(S\left(\theta e_{3}\right)\right) .
\end{aligned}
$$

Note that $F(\theta)$ is the matrix of rotation with angle $\varepsilon$ about the axis with unitary vector $(\sin \theta, \cos \theta, 0)^{T}$. A direct calculation yields

$$
F(\theta)=\left(\begin{array}{ccc}
c \varepsilon c^{2} \theta+s^{2} \theta & (1-c \varepsilon) c \theta s \theta & s \varepsilon c \theta \\
(1-c \varepsilon) c \theta s \theta & c^{2} \theta+s^{2} \theta c \varepsilon & -s \varepsilon s \theta \\
-s \varepsilon c \theta & s \varepsilon s \theta & c \varepsilon
\end{array}\right)
$$

where $s$ and $c$ denote the sin and $\cos$ functions, respectively.

Lemma 4: The function $F$ given by (32) is a transverse function for any $\varepsilon \in(0,2 \pi)$.

Proof: From (31)

$$
\begin{aligned}
\frac{\partial F}{\partial \theta}= & \exp \left(S\left(-\theta e_{3}\right)\right) S\left(-e_{3}\right) \exp \left(S\left(\varepsilon e_{2}\right)\right) \exp \left(S\left(\theta e_{3}\right)\right) \\
& +\exp \left(S\left(-\theta e_{3}\right)\right) \exp \left(S\left(\varepsilon e_{2}\right)\right) \exp \left(S\left(\theta e_{3}\right)\right) S\left(e_{3}\right)
\end{aligned}
$$

Using the fact that $R^{T} S(\omega) R=S\left(R^{T} \omega\right)$, for any rotation matrix $R$ and any vector $\omega \in \mathbb{R}^{3}$, we deduce from (33) that

$$
\frac{\partial F}{\partial \theta}=F S(\omega)
$$

with $\omega=\left(\mathrm{I}_{3 \times 3}-\exp \left(S\left(-\theta e_{3}\right)\right) \exp \left(S\left(-\varepsilon e_{2}\right)\right)\right) e_{3}$ and $\mathrm{I}_{3 \times 3} \in \mathbb{R}^{3 \times 3}$ the identity matrix. A direct calculation yields $\omega=(\cos \theta \sin \varepsilon,-\sin \theta \sin \varepsilon, 1-\cos \varepsilon)^{T}$. Since the transversality condition is equivalent to the third component of $\omega$ being different from zero, the proof follows. Q.E.D.

For the determination of a control expression one can use (19). This yields

$$
\begin{aligned}
& F(\theta)\left(S\left(e_{1}\right) u_{1}+S\left(e_{2}\right) u_{2}-S(\omega) \dot{\theta}\right) \\
& =F(\theta) R^{T} Z\left(R F(\theta)^{T}\right) F(\theta)
\end{aligned}
$$

or, equivalently

$$
S\left(e_{1} u_{1}+e_{2} u_{2}-\omega \dot{\theta}\right)=R^{T} Z\left(R F(\theta)^{T}\right) F(\theta) .
$$

This control is a practical stabilizer of the identity matrix provided that the v.f. $Z$ asymptotic stabilizes the identity matrix. A well known possible choice is $Z(R)=R S(-k r)$, with $k>0$ and $r \in \mathbb{R}^{3}$ the rotation vector associated with the rotation matrix $R$, i.e., the vector with smallest Euclidean norm (corresponding to the angle of rotation) such that $R=\exp S(r)$. The domain $\mathcal{D}$ in which this v.f. is differentiable and exponentially stabilizes the identity matrix is $\mathbf{S O}(3)$ minus the set of rotation matrices with angles of rotation equal to $\pi$. Using this expression of $Z$ in the previous equality yields

$$
\begin{aligned}
& S\left(e_{1} u_{1}+e_{2} u_{2}-\omega \dot{\theta}\right) \\
& \quad=F(\theta)^{T} S(-k z(\theta, r)) F(\theta) \\
& \quad=S\left(-k F(\theta)^{T} z(\theta, r)\right)
\end{aligned}
$$

with $z(\theta, r) \in \mathbb{R}^{3}$ denoting the rotation vector associated with the matrix $R F(\theta)^{T}$, i.e., such that $R F(\theta)^{T}=\exp S(z(\theta, r))$. A practical stabilizer is thus given by

$$
e_{1} u_{1}+e_{2} u_{2}-\omega \dot{\theta}=-k F(\theta)^{T} z(\theta, r)
$$

or, equivalently — after simple calculations—by

$$
\left(\begin{array}{c}
u_{1} \\
u_{2} \\
-\dot{\theta}
\end{array}\right)=-k\left(\begin{array}{ccc}
1 & 0 & -\frac{\omega_{1}}{\omega_{3}}(\theta) \\
0 & 1 & -\frac{\omega_{2}}{\omega_{3}}(\theta) \\
0 & 0 & \frac{1}{\omega_{3}}
\end{array}\right) F(\theta)^{T} z(\theta, r) .
$$

The domain of attraction in $\mathbf{S O}(\mathbf{3})$ contains the set of rotation matrices with angles of rotation smaller than $(\pi-\varepsilon)$.

\section{Unicycle}

Kinematic equations of the unicycle are

$$
\dot{g}=u_{1} X_{1}(g)+u_{2} X_{2}
$$

with $g=(x, y, \alpha)^{T}, X_{1}(g)=(\cos \alpha, \sin \alpha, 0)^{T}$, and $X_{2}=$ $(0,0,1)^{T}$. For stabilization purposes, this system is often transformed, via a diffeomorphic change of coordinates and new control variables, to the three dimensional chained system with two inputs. The solution described in Section VII-A then applies directly. However, the preliminary transformation into a 
chained system is not necessary, and the control design can be performed easily by using natural system coordinates. This is possible because, as this is well known, the above equations define a left-invariant control system on the Lie group $\mathbb{R}^{2} \times \mathbf{S}^{\mathbf{1}}$. The group operation is given by

$$
\left.g_{1} g_{2}=\left(\begin{array}{c}
x_{1} \\
y_{1}
\end{array}\right)+R\left(\alpha_{1}\right)\left(\begin{array}{l}
x_{2} \\
y_{2}
\end{array}\right)\right)
$$

with $R(\alpha)$ the rotation matrix in $\mathbb{R}^{2}$ of angle $\alpha$. The v.f. $X_{1}, X_{2}$, and $\left[X_{1}, X_{2}\right]$, form a graded basis of $\operatorname{Lie}\left(X_{1}, X_{2}\right)$, with $r=(1,1,2)$. A transverse function $f$ can be obtained by using (9)-(10), or the more general expression (9)-(12), i.e.,

$$
f(\theta)=\exp \left(\varepsilon_{1} \sin \theta X_{1}+\varepsilon_{2} \cos \theta X_{2}\right) .
$$

After simple calculations, using (35) and the expressions of the v.f. $X_{1}$ and $X_{2}$

$$
f(\theta)=\left(\begin{array}{c}
\varepsilon_{1} \sin \theta \frac{\sin \left(\varepsilon_{2} \cos \theta\right)}{\varepsilon_{2} \cos \theta} \\
\varepsilon_{1} \sin \theta \frac{1-\cos \left(\varepsilon_{2} \cos \theta\right)}{\varepsilon_{2} \cos \theta} \\
\varepsilon_{2} \cos \theta
\end{array}\right) .
$$

The transversality condition is equivalent to the fact that the matrix

$$
H(\theta)=\left(\begin{array}{lll}
X_{1}(f(\theta)) & X_{2} & \frac{\partial f}{\partial \theta}(\theta)
\end{array}\right)
$$

is invertible for any $\theta$. Using the expressions of $X_{1}, X_{2}$, and (36), one obtains

$$
\operatorname{det} H(\theta)=\varepsilon_{1} \varepsilon_{2} \frac{1-\cos \left(\varepsilon_{2} \cos \theta\right)}{\left(\varepsilon_{2} \cos \theta\right)^{2}}
$$

so that the transversality condition is satisfied for any $\left(\varepsilon_{1}, \varepsilon_{2}\right) \in$ $\left(\mathbb{R}^{+}-\{0\}\right)^{2}$. Another transverse function is obtained by using the control v.f. of the homogeneous approximation of system (34) which, in the present case, is the chained system of dimension three (up to a reordering of the state variables). Application of (9)-(12) then yields

$$
f(\theta)=\left(\varepsilon_{1} \sin \theta, \varepsilon_{1} \varepsilon_{2} \frac{\sin 2 \theta}{4}, \varepsilon_{2} \cos \theta\right)^{T} .
$$

One can verify that the transversality condition is satisfied with this function for $\varepsilon_{1} \neq 0$ and $\varepsilon_{2} \in(0, \pi / 2)$. From (35)

$$
\begin{aligned}
z & \left.\triangleq g f^{-1}=\left(\begin{array}{l}
x \\
y
\end{array}\right)-R\left(\alpha-f_{3}\right)\left(\begin{array}{l}
f_{1} \\
f_{2}
\end{array}\right)\right) \\
\alpha l_{g_{1}}\left(g_{2}\right) & =\left(\begin{array}{cc}
R\left(\alpha_{1}\right) & 0 \\
0 & 1
\end{array}\right)
\end{aligned}
$$

and

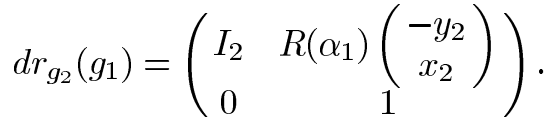

A practical stabilizer of $g=0$ is then given by (21) after choosing a v.f. $Z$ which asymptotically stabilizes the origin of $\dot{z}=Z(z)$. One can take, for example, $Z(z) \triangleq K z$, with $K$ any Hurwitz stable matrix, in order to achieve exponential stability.

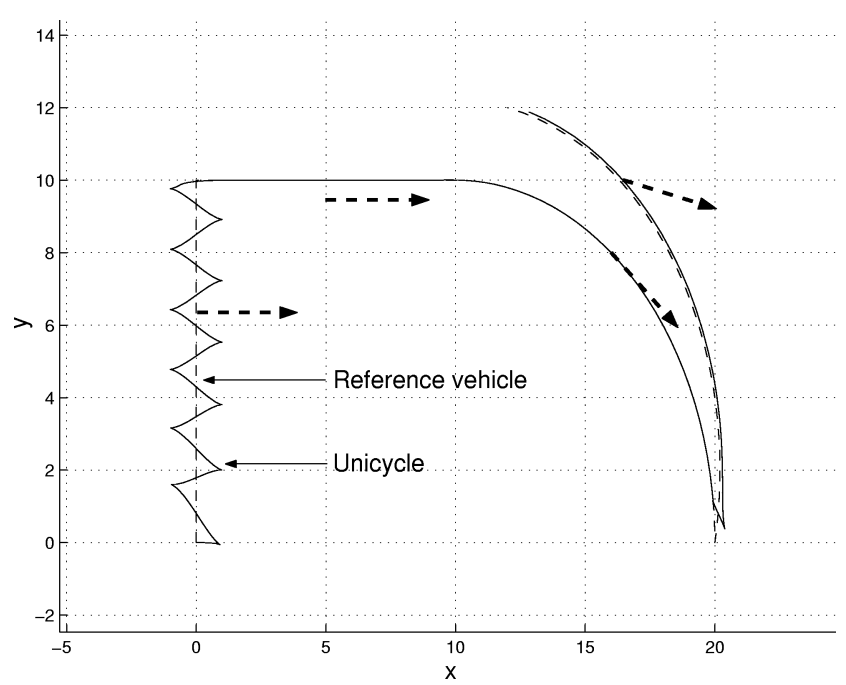

Fig. 1. Unicycle's and reference vehicle's $(x, y)$ trajectories.

We next report simulation results about the tracking of a reference vehicle by the unicycle. The posture of the reference vehicle is given by $g_{r}=\left(x_{r}, y_{r}, \alpha_{r}\right)^{T}$ and the control is determined so as to stabilize $z=\tilde{g} f^{-1}$ to zero, with

$$
\tilde{g}=g_{r}^{-1} g=\left(\begin{array}{c}
R\left(-\alpha_{r}\right)\left(\begin{array}{c}
x-x_{r} \\
y-y_{r}
\end{array}\right) \\
\alpha-\alpha_{r}
\end{array}\right)
$$

representing the tracking error. Such a control is given by (21) with, of course, $g$ replaced by $\tilde{g}$. The chosen transverse function is given by (37) with $\varepsilon_{1}=1$ and $\varepsilon_{2}=0.5$. The chosen stabilizing v.f. $Z$ is defined by $Z(z)=-1 / 2\left(z_{1}, z_{2}, z_{3}\right)^{T}$. The expression of $P(\tilde{g}, t)$ can be obtained either by application of (23), or by direct computation of $\dot{\tilde{g}}$. By setting

$$
\dot{g}_{r}=u_{1}^{r} X_{1}\left(g_{r}\right)+u_{2}^{r} X_{2}+u_{3}^{r}\left[X_{2}, X_{1}\right]\left(g_{r}\right)
$$

one obtains

$$
P(\tilde{g}, t)=-u_{1}^{r}\left(\begin{array}{l}
1 \\
0 \\
0
\end{array}\right)-u_{2}^{r}\left(\begin{array}{c}
-\tilde{g}_{2} \\
\tilde{g}_{1} \\
1
\end{array}\right)-u_{3}^{r}\left(\begin{array}{l}
0 \\
1 \\
0
\end{array}\right) .
$$

All terms in the feedback expression (21) are now defined. For the simulation, the control inputs $u_{1}^{r}, u_{2}^{r}$, and $u_{3}^{r}$ for the reference vehicle, on the time interval $[0,40] s$, are given by

$$
\begin{aligned}
& t \in[0,10): u_{1}^{r}=0 \quad u_{2}^{r}=0 \quad u_{3}^{r}=1 \\
& t \in[10,20): u_{1}^{r}=1 \quad u_{2}^{r}=0 \quad u_{3}^{r}=0 \\
& t \in[20,30): u_{1}^{r}=\pi / 2 \quad u_{2}^{r}=-\pi / 20 \quad u_{3}^{r}=0 \\
& t \in[30,40]: u_{1}^{r}=-\frac{\pi}{2} \quad u_{2}^{r}=\frac{\pi}{20} \quad u_{3}^{r}=2 u_{2}^{r} .
\end{aligned}
$$

Note that for $t \in[0,10)$ and $t \in[30,40]$, the reference trajectory is not feasible by the unicycle (since $u_{3}^{r} \neq 0$ ). Fig. 1 shows the trajectories of the unicycle and the reference vehicle in the plane, with the dashed arrows indicating the orientation of the reference vehicle. Fig. 2 shows the time evolution of the components of the tracking vector $\tilde{g}$ and the unicycle's control inputs $u_{1}$ and $u_{2}$. We remark that the unicycle does not oscillate when the reference vehicle follows portions of "feasible" trajectories, 

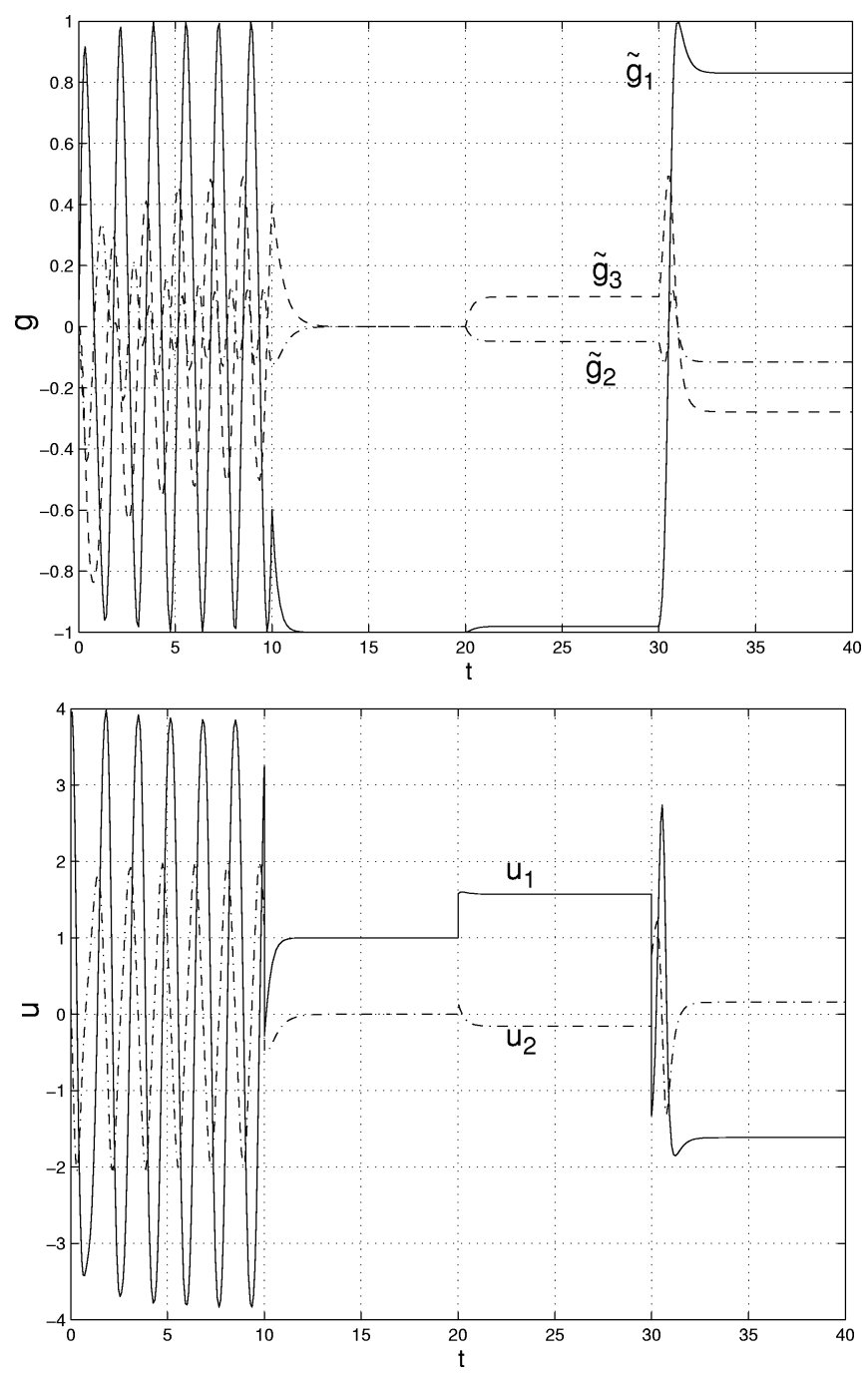

Fig. 2. Tracking errors and unicycle's control inputs.

as in the case of a straight line, when $t \in[10,20)$, and an arc of circle, when $t \in[20,30)$. The simulation further shows that, as mentioned in Section I, oscillations are not systematic when the reference trajectory is not feasible. This is illustrated by the portion of trajectory corresponding to $t \in[30,40]$. Sustained oscillations only occur during the first phase corresponding to $t \in[0,10)$, when practical tracking of the reference vehicle with the "precision" specified by $\left(\varepsilon_{1}, \varepsilon_{2}\right)$ requires them.

\section{APPENDIX A}

PROOFS OF LEMMAS 1-3

The following technical claims are used in the proofs of Lemmas 1 and 2.

Claim 1: Let $Y$ and $Z$ denote two time-dependent left-invariant v.f. on $G$, and $\sigma, \tau$ solutions of $\dot{\sigma}=Y(\sigma, t)$ and $\dot{\tau}=Z(\tau, t)$ respectively. Then $\nu \triangleq \sigma \tau$ is a solution of $\dot{\nu}=\operatorname{Ad}\left(\tau^{-1}\right) Y(\nu, t)+Z(\nu, t)$.

The proof follows by classical calculus on Lie groups.

Claim 2: Let $\left\{X_{1}, \ldots, X_{n}\right\}$ denote a graded basis of the Lie algebra $\mathfrak{g}$ of a Lie group $G$. Let $\lambda, \rho, q \in\{1, \ldots, n\}$ and $s \in \mathbb{N}$.
Then, there exist analytic functions $g_{1}, \ldots, g_{n}$ such that, for any $\alpha_{\lambda}, \alpha_{\rho} \in \mathbb{R}$

$$
\sum_{j=s}^{\infty} \frac{1}{j !}\left(\operatorname{ad}^{j}\left(\alpha_{\lambda} X_{\lambda}+\alpha_{\rho} X_{\rho}\right), X_{q}\right)=\sum_{k=1}^{n} g_{k}\left(\alpha_{\lambda}, \alpha_{\rho}\right) X_{k} .
$$

Furthermore, if $\alpha_{\lambda}:(\varepsilon, \theta) \in \mathbb{R} \times \mathbb{T} \longmapsto \alpha_{\lambda}(\varepsilon, \theta)$ and $\alpha_{\rho}:(\varepsilon, \theta) \in \mathbb{R} \times \mathbb{T} \longmapsto \alpha_{\rho}(\varepsilon, \theta)$ denote analytic functions such that $\alpha_{\lambda}=\mathrm{O}\left(|\varepsilon|^{r_{\lambda}}\right)$ and $\alpha_{\rho}=\mathrm{O}\left(|\varepsilon|^{r_{\rho}}\right)$, then $g_{k}\left(\alpha_{\lambda}, \alpha_{\rho}\right)$ is an analytic function and $g_{k}\left(\alpha_{\lambda}, \alpha_{\rho}\right)=$ $\mathrm{O}\left(|\varepsilon|^{\max \left\{s \min \left\{r_{\lambda}, r_{\rho}\right\}, r_{k}-r_{q}\right\}}\right)$.

Proof: Let $c_{i, j}^{k}$ denote the structural constants associated with $X_{1}, \ldots, X_{n}$, i.e.,

$$
\left[X_{i}, X_{j}\right]=\sum_{k} c_{i, j}^{k} X_{k}
$$

From Definition 1, one deduces that $r_{k}>r_{i}+r_{j} \Longrightarrow c_{i, j}^{k}=0$. From this fact, one obtains by induction on $j$

$$
\begin{aligned}
& \left.\operatorname{(ad}^{j}\left(\alpha_{\lambda} X_{\lambda}+\alpha_{\rho} X_{\rho}\right), X_{q}\right) \\
& =\sum_{\eta=0}^{j} \alpha_{\lambda}^{\eta} \alpha_{\rho}^{j-\eta} \sum_{r_{k_{j}} \leq \eta r_{\lambda}+(j-\eta) r_{\rho}+r_{q}} a_{j, k_{j}}^{\eta} X_{k_{j}}
\end{aligned}
$$

with

$$
\left|a_{j, k_{j}}^{\eta}\right| \leq(2 M n)^{j} \quad \text { and } \quad M \triangleq \max \left|c_{i, j}^{k}\right| .
$$

Therefore

$$
\begin{aligned}
& \sum_{j=s}^{\infty} \frac{1}{j !}\left(\operatorname{ad}^{j}\left(\alpha_{\lambda} X_{\lambda}+\alpha_{\rho} X_{\rho}\right), X_{q}\right) \\
& \quad=\sum_{j=s}^{\infty} \frac{1}{j !} \sum_{\eta=0}^{j} \alpha_{\lambda}^{\eta} \alpha_{\rho}^{j-\eta} \sum_{r_{k_{j}} \leq \eta r_{\lambda}+(j-\eta) r_{\rho}+r_{q}} a_{j, k_{j}}^{\eta} X_{k_{j}} \\
& \quad=\sum_{k=1}^{n} \sum_{j \geq s} \frac{\alpha_{\lambda}^{\eta} \alpha_{\rho}^{j-\eta}}{j !} \sum_{j \leq \eta \leq j, \eta r_{\lambda}+(j-\eta) r_{\rho} \geq r_{k}-r_{q}} X_{k} \\
& \quad=\sum_{k=1}^{n} g_{k}\left(\alpha_{\lambda}, \alpha_{\rho}\right) X_{k}
\end{aligned}
$$

with

$$
g_{k}\left(\alpha_{\lambda}, \alpha_{\rho}\right)=\sum_{j \geq s} \sum_{0 \leq \eta \leq j, \eta r_{\lambda}+(j-\eta) r_{\rho} \geq r_{k}-r_{q}} \frac{\alpha_{\lambda}^{\eta} \alpha_{\rho}^{j-\eta}}{j !} a_{j, k}^{\eta} .
$$

It follows from (38) that each $g_{k}$ is an analytic function of $\alpha_{\lambda}$ and $\alpha_{\rho}$. Furthermore, if $\alpha_{\lambda}=\mathrm{O}\left(|\varepsilon|^{r_{\lambda}}\right)$ and $\alpha_{\rho}=\mathrm{O}\left(|\varepsilon|^{r_{\rho}}\right)$ are analytic functions of $\varepsilon$ and $\theta$, then $g_{k}\left(\alpha_{\lambda}, \alpha_{\rho}\right)$ is analytic and it follows, by considering the term of lowest order in (39), that

$$
g_{k}\left(\alpha_{\lambda}, \alpha_{\rho}\right)=\mathrm{O}\left(|\varepsilon|^{\max \left\{s \min \left\{r_{\lambda}, r_{\rho}\right\}, r_{k}-r_{q}\right\}}\right) .
$$

Note that the equality is uniform w.r.t. $\theta$ because $g_{k}\left(\alpha_{\lambda}, \alpha_{\rho}\right)$ is periodic w.r.t $\theta$. 
Proof of Lemma 1: In order to lighten the notation, we denote by $f_{j}^{\prime}$ the derivative of $f_{j}$ w.r.t $\theta_{j}$. We will also use $X_{\lambda}$ for $X_{\lambda(j)}, X_{\rho}$ for $X_{\rho(j)}$, and $X$ for $X_{j}$, and denote by $\alpha_{\lambda}$ and $\alpha \rho$ the functions defined by

$$
\begin{aligned}
& \alpha_{\lambda}\left(\varepsilon_{j}, \theta_{j}\right)=\varepsilon_{j}^{r_{\lambda(j)}} \sin \theta_{j} \\
& \alpha_{\rho}\left(\varepsilon_{j}, \theta_{j}\right)=\varepsilon_{j}^{r_{\rho(j)}} \cos \theta_{j} .
\end{aligned}
$$

With this notation, $f_{j}=\exp \left(\alpha_{\lambda} X_{\lambda}+\alpha_{\rho} X_{\rho}\right)$. In order to calculate $f_{j}^{\prime}$ we will use the following classical result ( $[15$, p. 105], for example):

$$
\frac{d}{d s} \exp (X+s Y)_{\mid s=0}=(\phi(\operatorname{ad} X), Y)(\exp X)
$$

with $\phi$ the operator defined by

$$
\phi(z)=\sum_{j=0}^{\infty} \frac{(-1)^{j}}{(j+1) !} z^{j}\left(=\frac{1-e^{-z}}{z}\right) .
$$

Since $(d / d \theta) \exp X(\theta)=(d / d s) \exp \left(X(\theta)+s X^{\prime}(\theta)\right)_{\mid s=0}$, one obtains, by application of (41)

$$
\begin{aligned}
f_{j}^{\prime}= & \left(\phi\left(\operatorname{ad}\left(\alpha_{\lambda} X_{\lambda}+\alpha_{\rho} X_{\rho}\right)\right), \alpha_{\lambda}^{\prime} X_{\lambda}+\alpha_{\rho}^{\prime} X_{\rho}\right)\left(f_{j}\right) \\
= & \alpha_{\lambda}^{\prime} X_{\lambda}\left(f_{j}\right)+\alpha_{\rho}^{\prime} X_{\rho}\left(f_{j}\right) \\
& -\frac{1}{2}\left[\alpha_{\lambda} X_{\lambda}+\alpha_{\rho} X_{\rho}, \alpha_{\lambda}^{\prime} X_{\lambda}+\alpha_{\rho}^{\prime} X_{\rho}\right]\left(f_{j}\right) \\
& +\sum_{i=2}^{\infty} \frac{(-1)^{i}}{(i+1) !}\left(\operatorname{ad}^{i}\left(\alpha_{\lambda} X_{\lambda}+\alpha_{\rho} X_{\rho}\right),\right. \\
& \left.\alpha_{\lambda}^{\prime} X_{\lambda}+\alpha_{\rho}^{\prime} X_{\rho}\right)\left(f_{j}\right) \\
= & \alpha_{\lambda}^{\prime} X_{\lambda}\left(f_{j}\right)+\alpha_{\rho}^{\prime} X_{\rho}\left(f_{j}\right)-\frac{\left(\alpha_{\lambda} \alpha_{\rho}^{\prime}-\alpha_{\rho} \alpha_{\lambda}^{\prime}\right)}{2} \\
& \times\left[X_{\lambda}, X_{\rho}\right]\left(f_{j}\right) \\
& +\left(\alpha_{\lambda} \alpha_{\rho}^{\prime}-\alpha_{\rho} \alpha_{\lambda}^{\prime}\right) \sum_{i=1}^{\infty} \frac{(-1)^{i+1}}{(i+2) !} \\
& \times\left(\operatorname{ad}^{i}\left(\alpha_{\lambda} X_{\lambda}+\alpha_{\rho} X_{\rho}\right),\left[X_{\lambda}, X_{\rho}\right]\right)\left(f_{j}\right) .
\end{aligned}
$$

In view of (40), and since $X=\left[X_{\lambda}, X_{\rho}\right]$ (by Definition 1), the aforementioned equality yields

$$
\begin{aligned}
f_{j}^{\prime}= & \alpha_{\lambda}^{\prime} X_{\lambda}\left(f_{j}\right)+\alpha_{\rho}^{\prime} X_{\rho}\left(f_{j}\right) \\
& +\frac{\varepsilon_{j}^{r_{j}}}{2} X\left(f_{j}\right)-\varepsilon_{j}^{r_{j}} \sum_{i=1}^{\infty} \frac{(-1)^{i+1}}{(i+2) !} \\
& \times\left(\operatorname{ad}^{i}\left(\alpha_{\lambda} X_{\lambda}+\alpha_{\rho} X_{\rho}\right), X\right)\left(f_{j}\right) .
\end{aligned}
$$

By application of Claim 2

$$
\begin{aligned}
\varepsilon_{j}^{r_{j}} \sum_{i=1}^{\infty} \frac{(-1)^{i+1}}{(i+2) !}\left(\operatorname{ad}^{i}\left(\alpha_{\lambda} X_{\lambda}+\alpha_{\rho} X_{\rho}\right), X\right) & \\
& =\sum_{k=1}^{n} g_{k}\left(\alpha_{\lambda}, \alpha_{\rho}\right) X_{k}
\end{aligned}
$$

for some analytic functions $g_{k}(k=1, \ldots, n)$ such that

$$
g_{k}\left(\alpha_{\lambda}, \alpha_{\rho}\right)=O\left(\left|\varepsilon_{j}\right|^{\max \left\{r_{j}+\min \left\{r_{\rho}, r_{\lambda}\right\}, r_{k}\right\}}\right) .
$$

Lemma 1 follows by using (43)-(44) in (42).
Proof of Lemma 2: From Claim 1, and relations (9) and (13), one deduces that

$$
\frac{\partial f}{\partial \theta_{j}}=\sum_{k=1}^{n} v_{k, j} \operatorname{Ad}\left(f_{m+1}^{-1} \ldots f_{j-1}^{-1}\right) X_{k}(f) .
$$

From the fact that $\operatorname{Ad}\left(g_{1} g_{2}\right)=\operatorname{Ad}\left(g_{1}\right) \operatorname{Ad}\left(g_{2}\right)$ and (10)

$$
\begin{aligned}
\operatorname{Ad} & \left(f_{m+1}^{-1} \cdots f_{j-1}^{-1}\right) \\
& =\prod_{p=m+1}^{j-1} \operatorname{Ad}\left(f_{p}^{-1}\right) \\
& =\prod_{p=m+1}^{j-1} \operatorname{Ad}\left(\exp \left(-\alpha_{\lambda(p)} X_{\lambda(p)}-\alpha_{\rho(p)} X_{\rho(p)}\right)\right)
\end{aligned}
$$

where $\alpha_{\lambda(p)}$ and $\alpha_{\rho(p)}$ are defined according to (40). By application of Claim 2, for any $\lambda, \rho, q=1, \ldots, n$

$$
\operatorname{Ad}\left(\exp \left(-\alpha_{\lambda} X_{\lambda}-\alpha_{\rho} X_{\rho}\right)\right) X_{q}=X_{q}+\sum_{i=1}^{n} h_{\lambda, \rho, q}^{i} X_{i}
$$

for some analytic functions $h_{\lambda, \rho, q}^{i}$. Moreover, if $\alpha_{\lambda}=$ $\mathrm{O}\left(|\varepsilon|^{r_{\lambda}}\right)$ and $\alpha_{\rho}=\mathrm{O}\left(|\varepsilon|^{r_{\rho}}\right)$ are analytic functions then, $h_{\lambda, \rho, q}^{i}\left(\alpha_{\lambda}, \alpha_{\rho}\right)=\mathrm{O}\left(|\varepsilon|^{\max \left\{1, r_{i}-r_{q}\right\}}\right)$. By applying this property recursively, one deduces from (46) that

$$
\operatorname{Ad}\left(f_{m+1}^{-1} \ldots f_{j-1}^{-1}\right) X_{k}=X_{k}+\sum_{i=1}^{n} g_{j, k}^{i} X_{i}
$$

for some analytic functions $g_{j, k}^{i}$ which depend on $\varepsilon_{m+1} \ldots$, $\varepsilon_{j-1}, \theta_{m+1}, \ldots, \theta_{j-1}$, and are such that

$$
g_{j, k}^{i}=\mathrm{O}\left(\left|\bar{\varepsilon}_{j-1}\right|^{\max \left\{1, r_{i}-r_{k}\right\}}\right)
$$

(recall the notation $\bar{\varepsilon}_{k}=\left(\varepsilon_{m+1}, \ldots, \varepsilon_{k}\right)$ ). From (47)

$$
\begin{aligned}
\sum_{k=1}^{n} v_{k, j} \operatorname{Ad}\left(f_{m+1}^{-1} \cdots f_{j-1}^{-1}\right) & X_{k}(f) \\
= & \sum_{i=1}^{n}\left(v_{i, j}+\sum_{k=1}^{n} v_{k, j} g_{j, k}^{i}\right) X_{i}(f)
\end{aligned}
$$

so that, by (45), (15) is satisfied with

$$
a_{i, j} \triangleq v_{i, j}+\sum_{k=1}^{n} v_{k, j} g_{j, k}^{i}=A+B+C
$$

where

$$
\begin{aligned}
A & =\sum_{r_{k} \leq r_{i}} v_{k, j} g_{j, k}^{i} \quad B=v_{i, j} \\
C & =\sum_{r_{k}>r_{i}} v_{k, j} g_{j, k}^{i} .
\end{aligned}
$$

Lemma 2 follows from this decomposition. Indeed, from Lemma 1 and (48), $A, B$, and $C$ in (50) are $\mathrm{O}\left(\left|\bar{\varepsilon}_{j}\right|^{r_{i}}\right)$. This proves the first relation of (16).

Concerning the case where $i<j$ and $r_{i}=r_{j}, A$ vanishes at $\bar{\varepsilon}_{j-1}=0$ because of (48). This accounts for the sum in the right-hand side of equality (16) (up to higher-order terms). Now, because of (14), $B=\mathrm{o}\left(\left|\varepsilon_{j}\right|^{r_{i}}\right)=\mathrm{o}\left(\left|\bar{\varepsilon}_{j}\right|^{r_{i}}\right)$ and $C=$ Q.E.D. $\mathrm{O}\left(\left|\varepsilon_{j}\right|^{r_{k}}\right)=\mathrm{o}\left(\left|\varepsilon_{j}\right|^{r_{i}}\right)=\mathrm{o}\left(\left|\bar{\varepsilon}_{j}\right|^{r_{i}}\right)$ too. 
For the last case, $i=j, A$ accounts for the sum in the right-hand side of equality (16) and $C=\mathrm{o}\left(\left|\bar{\varepsilon}_{j}\right|^{r_{j}}\right)$, as in the previous case. From Lemma $1, B$ accounts for the term $\varepsilon_{j}^{r_{j}} / 2$.

Q.E.D.

Proof of Lemma 3: The notation $\bar{r}_{k} \triangleq r_{m+1}+\cdots+r_{k}$ is used in this proof. The lemma is a direct consequence of the following property which will be proved by induction:

$$
\begin{aligned}
& \forall k=m+1, \ldots, n \quad \exists \bar{\eta}_{k} \in \mathbb{R}^{k-m} \quad \exists \alpha_{k}>0: \\
& \bar{\varepsilon}_{k}=\varepsilon_{k} \bar{\eta}_{k} \Longrightarrow \mathrm{D}_{k} \geq \alpha_{k} \bar{\varepsilon}_{k}^{\bar{r}_{k}}+\mathrm{o}\left(\left|\varepsilon_{k}\right|^{\bar{r}_{k}}\right)
\end{aligned}
$$

with $\mathrm{D}_{k}$ the function defined by

$$
\mathrm{D}_{k}(\theta) \triangleq \operatorname{det}\left(a_{i, j}(\theta)\right)_{i, j=m+1, \ldots, k} .
$$

Let us first prove (51) for $k=m+1$. From Lemma 2, $a_{m+1, m+1}=(1 / 2) \varepsilon_{m+1}^{r_{m+1}}+\mathrm{o}\left(\left|\varepsilon_{m+1}\right|^{r_{m+1}}\right)$. Since $\mathrm{D}_{m+1}=a_{m+1, m+1}$ and $\bar{r}_{m+1}=r_{m+1}$, (51) follows with $\bar{\eta}_{m+1}=1$ and $\alpha_{m+1}=1 / 2$.

Let us now prove that if (51) is satisfied for $k$ (with $m+1 \leq$ $k<n$ ), then it is also satisfied for $k+1$. Let

$$
\bar{\varepsilon}_{k+1}=\varepsilon_{k+1} \bar{\eta}_{k+1} \quad \text { with } \bar{\eta}_{k+1}=\left(\gamma_{k+1} \bar{\eta}_{k}, 1\right) .
$$

In these equalities, $\bar{\eta}_{k}$ denotes the vector involved in (51), whereas $\gamma_{k+1}$ is a scalar design parameter whose value will be specified below. By (52), $\bar{\varepsilon}_{k}=\left(\varepsilon_{k+1} \gamma_{k+1}\right) \bar{\eta}_{k}$. Therefore, one easily obtains from Lemma 2 the following equalities:

$$
\begin{aligned}
& \forall i \quad \forall q<k+1 \quad a_{i, q}=\mathrm{O}\left(\left|\varepsilon_{k+1} \gamma_{k+1}\right|^{r_{i}}\right)
\end{aligned}
$$

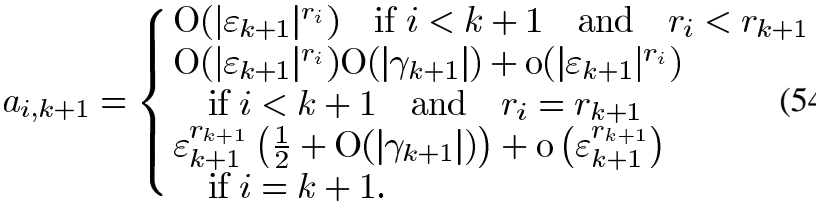

By definition

$$
\mathrm{D}_{k+1}=a_{k+1, k+1} \mathrm{D}_{k}+\sum_{i=1}^{k} a_{i, k+1} \operatorname{Cof}\left(a_{i, k+1}\right)
$$

where $\operatorname{Cof}\left(a_{i, k+1}\right)$ denotes the cofactor of $a_{i, k+1}$. From (53)

$$
\operatorname{Cof}\left(a_{i, k+1}\right)=\mathrm{O}\left(\left|\varepsilon_{k+1} \gamma_{k+1}\right|^{\bar{r}_{k+1}-r_{i}}\right) .
$$

From the induction hypothesis (51), and the fact that, by a recursive application of (52), $\varepsilon_{k}=\varepsilon_{k+1} \gamma_{k+1}$

$$
\mathrm{D}_{k} \geq \alpha_{k}\left(\varepsilon_{k+1} \gamma_{k+1}\right)^{\bar{r}_{k}}+\mathrm{o}\left(\left|\varepsilon_{k+1} \gamma_{k+1}\right|^{\bar{r}_{k}}\right) .
$$

By assuming that $\gamma_{k+1}, \varepsilon_{k+1}>0$ are small enough so that $a_{k+1, k+1}>0$, one deduces from (54) and (57) that

$$
\begin{aligned}
a_{k+1, k+1} D_{k} \geq \alpha_{k} \gamma_{k+1}^{\bar{r}_{k}}\left(\frac{1}{2}+\mathrm{O}\left(\left|\gamma_{k+1}\right|\right)\right) & \varepsilon_{k+1}^{\bar{r}_{k+1}} \\
& +\mathrm{o}\left(\left|\varepsilon_{k+1}\right|^{\bar{r}_{k+1}}\right) .
\end{aligned}
$$

From (54) and (56), for any $i=1, \ldots, k$

$$
\begin{array}{r}
a_{i, k+1} \operatorname{Cof}\left(a_{i, k+1}\right)=\varepsilon_{k+1}^{\bar{r}_{k+1}} \mathrm{O}\left(\left|\gamma_{k+1}\right|^{\bar{r}_{k}+1}\right) \\
+\mathrm{o}\left(\left|\varepsilon_{k+1}\right|^{\bar{r}_{k+1}}\right)
\end{array}
$$

Therefore, from (55), (58), and (59)

$\mathrm{D}_{k+1} \geq \alpha_{k} \gamma_{k+1}^{\bar{r}_{k}}\left(\frac{1}{2}+\mathrm{O}\left(\left|\gamma_{k+1}\right|\right)\right) \varepsilon_{k+1}^{\bar{r}_{k+1}}$

$$
+\mathrm{o}\left(\left|\varepsilon_{k+1}\right|^{\bar{r}_{k+1}}\right)
$$

Then, (51) for $k+1$ follows by choosing $\gamma_{k+1}>0$ small enough so as to ensure that

$$
\alpha_{k+1} \triangleq \frac{1}{4} \alpha_{k} \gamma_{k+1}^{\bar{r}_{k}} \leq \alpha_{k} \gamma_{k+1}^{\bar{r}_{k}}\left(\frac{1}{2}+\mathrm{O}\left(\left|\gamma_{k+1}\right|\right)\right)
$$

is different from zero. Thus, we have proved by induction the existence of $n-m$ numbers $\gamma_{m+1}, \ldots, \gamma_{n}$ (with $\gamma_{m+1}=1$ ) such that choosing [see (52)]

$$
\bar{\varepsilon}_{n}=\varepsilon_{n}\left(\prod_{i=m+2}^{n} \gamma_{i}, \ldots, \gamma_{n-1} \gamma_{n}, \gamma_{n}, 1\right)
$$

yields $\forall \theta, \mathrm{D}_{n}(\theta) \neq 0$, provided that $\varepsilon_{n}$ is itself chosen small enough. This is precisely the result of Lemma 3. Q.E.D.

\section{REFERENCES}

[1] A. Astolfi, "Discontinuous control of nonholonomic systems," Syst. Control Letters, vol. 27, pp. 37-45, 1996.

[2] M. K. Bennani and P. Rouchon, "Robust stabilization of flat and chained systems," in Proc. Euro. Control Conf., 1995, pp. 2642-2646.

[3] A. M. Bloch and S. Drakunov, "Stabilization and tracking in the nonholonomic integrator via sliding modes," Syst. Control Lett., vol. 29, pp. 91-99, 1996.

[4] A. M. Bloch, M. Reyhanoglu, and N. H. McClamroch, "Control and stabilization of nonholonomic dynamic systems," IEEE Trans. Automat. Contr., vol. 37, pp. 1746-1757, 1992.

[5] R. W. Brockett, "Asymptotic stability and feedback stabilization," in Differential Geometric Control Theory, R. S. Millman, R. W. Brockett, and H. J. Sussmann, Eds. Boston, MA: Birkaüser, 1983.

[6] F. Bullo, N. H. Leonard, and A. D. Lewis, "Controllability and motion algorithms for underactuated lagrangian systems on Lie groups," IEEE Trans. Automat. Contr., vol. 45, pp. 1437-1454, 2000.

[7] C. Canudas de Wit, B. Siciliano, and G. Bastin, Eds., Theory of Robot Control. New York: Springer-Verlag, 1996.

[8] C. Canudas de Wit and O. J. Sørdalen, "Exponential stabilization of mobile robots with nonholonomic constraints," IEEE Trans. Automat. Contr., vol. 37, pp. 1791-1797, Nov. 1992.

[9] W. L. Chow, "Uber systeme von linearen partiellen differential-gleichungen erster ordnung," Math. Ann., vol. 117, pp. 98-105, 1939.

[10] J.-M. Coron, "Global asymptotic stabilization for controllable systems without drift," Math. Control, Sig., Syst., vol. 5, pp. 295-312, 1992.

[11] D. M. Dawson, J. Hu, and P. Vedagharba, "An adaptive control for a class of induction motor systems," in Proc. IEEE Conf. Decision Control, 1995, pp. 1567-1572.

[12] W. E. Dixon, D. M. Dawson, E. Zergeroglu, and F. Zhang, "Robust tracking and regulation control for mobile robots," Int. J. Robust Nonlinear Control, vol. 10, pp. 199-216, 2000.

[13] M. Fliess, J. Lévine, P. Martin, and P. Rouchon, "Flatness and defect of nonlinear systems: Introductory theory and examples," Int. J. Control, vol. 61, pp. 1327-1361, 1995.

[14] G. B. Folland, "On the Rothschild-Stein lifting theorem," Commun. Partial Differential Equat., vol. 2, pp. 165-191, 1977.

[15] S. Helgason, Differential Geometry, Lie Groups, and Symmetric Spaces. New York: Academic, 1978.

[16] H. Hermes, "Nilpotent and high-order approximations of vector field systems," SIAM Rev., vol. 33, pp. 238-264, 1991.

[17] Y. Kanayama, A. Nilipour, and C. Lelm, "A locomotion control method for autonomous vehicles," in Proc. IEEE ICRA, 1988, pp. 1315-1317.

[18] M. Kawski, "Nonlinear control and combinatorics of words," in Nonlinear Feedback and Optimal Control, B. Jakubczyk and W. Respondek, Eds. New York: Marcel Dekker, 1998.

[19] M. Kawski and H. J. Sussmann, "Noncommutative power series and formal Lie-algebraic techniques in nonlinear control theory," in Operators, Systems, and Linear Algebra, D. Pratzel-Wolters, U. Helmke, and E. Zerz, Eds. New York: Teubner, 1997, pp. 111-128. 
[20] G. Lafferriere and H. J. Sussmann, "A differential geometric approach to motion planning," in Nonholonomic Motion Planning, Z. Li and J. F. Canny, Eds. Norwell, MA: Kluwer, 1993.

[21] N. E. Leonard and P. S. Krishnaprasad, "Motion control of drift-free, left-invariant systems on Lie groups," IEEE Trans. Automat. Contr., vol. 40, pp. 1539-1554, 1995.

[22] W. Lin and P. Radom, "Recursive design of discontinuous controllers for uncertain driftless systems in power chained form," IEEE Trans. Automat. Contr., vol. 45, pp. 1886-1892, 2000.

[23] W. Liu, "An approximation algorithm for nonholonomic systems," SIAM J. Control Optim., vol. 35, pp. 1328-1365, 1997.

[24] D. A. Lizárraga, "Obstructions to the existence of universal stabilizers for smooth control systems," Math. Control, Sign, Syst., 2003, to be published.

[25] D. A. Lizárraga, P. Morin, and C. Samson, "Non-robustness of continuous homogeneous stabilizers for affine control systems," in Proc. IEEE Conf. Decision Control, 1999, pp. 855-860.

[26] M. Maini, P. Morin, J.-B. Pomet, and C. Samson, "On the robust stabilization of chained systems by continuous feedback," in Proc. IEEE Conf. Decision Control, 1999, pp. 3472-3477.

[27] P. Martin and P. Rouchon, "Feedback linearization of driftless systems," Math. Control, Sig., Syst., vol. 7, pp. 235-254, 1994.

[28] R. T. M'Closkey and R. M. Murray, "Exponential stabilization of driftless nonlinear control systems using homogeneous feedback," IEEE Trans. Automat. Contr., vol. 42, pp. 614-628, 1997.

[29] P. Morin, J.-B. Pomet, and C. Samson, "Design of homogeneous timevarying stabilizing control laws for driftless controllable systems via oscillatory approximation of Lie brackets in closed-loop," SIAM J. Conttol Optim., vol. 38, pp. 22-49, 1999.

[30] P. Morin and C. Samson, "Exponential stabilization of nonlinear driftless systems with robustness to unmodeled dynamics," Control Optim. Calculus Var, vol. 4, pp. 1-36, 1999.

[31] — " "Control of nonlinear chained systems. From the Routh-Hurwitz stability criterion to time-varying exponential stabilizers," IEEE Trans. Automat. Contr., vol. 45, pp. 141-146, 2000.

[32] - "Practical stabilization of a class of nonlinear systems. Application to chain systems and mobile robots," in Proc. IEEE Conf. Decision Control, 2000, pp. 2989-2994.

[33] _ - "Practical stabilization of driftless systems on lie groups," INRIA, Tech. Rep. 4294, 2001.

[34] - "A characterization of the Lie algebra rank condition by transverse periodic functions," SIAM J. Control Optim., vol. 40, pp. 1227-1249, 2002.

[35] R. M. Murray and S. S. Sastry, "Nonholonomic motion planning: Steering using sinusoids," IEEE Trans. Automat. Contr., vol. 38, pp. 700-716, 1993

[36] J.-B. Pomet, "Explicit design of time-varying stabilizing control laws for a class of controllable systems without drift," Syst. Control Lett., vol. 18, pp. 467-473, 1992.

[37] J.-B. Pomet and C. Samson, "Exponential stabilization of nonholonomic systems in power form," IFAC Symp. Robust Control Design, pp. 447-452, 1994

[38] L. P. Rothschild and E. M. Stein, "Hypoelliptic differential operators and nilpotent groups," Acta Math., vol. 137, pp. 247-320, 1976.
[39] C. Samson, "Velocity and torque feedback control of a nonholonomic cart," presented at the Int. Workshop Adaptative Nonlinear Control: Issues in Robotics, 1990.

[40] _ - "Control of chained systems. Application to path following and time-varying point-stabilization," IEEE Trans. Automat. Contr., vol. 40, pp. 64-77, Jan. 1995.

[41] O. J. Sørdalen and O. Egeland, "Exponential stabilization of nonholonomic chained systems," IEEE Trans. Automat. Contr., vol. 40, pp. 35-49, Jan. 1995.

[42] G. Stefani, "Polynomial approximations to control systems and local controllability," in Proc. IEEE Conf. Decision Control, 1985, pp. 33-38.

[43] H. J. Sussmann, "Lie brackets and local controllability: A sufficient condition for scalar-input systems," SIAM J. Control Optim., vol. 21, pp. 686-713, 1983

[44] H. J. Sussmann and W. Liu, "Limits of highly oscillatory controls and approximation of general paths by admissible trajectories," in Proc. IEEE Conf. Decision Control, 1991, pp. 437-442.

[45] A. R. Teel, R. M. Murray, and G. Walsh, "Nonholonomic control systems: From steering to stabilization with sinusoids," Int. J. Control, vol. 62, pp. 849-870, 1995.

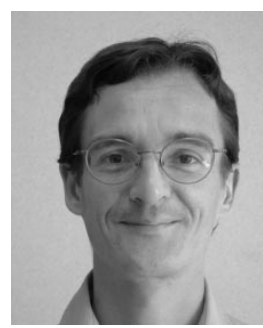

Pascal Morin was born in Avranches, France, in 1969. He received the Maitrise degree from Université Paris-Dauphine, Paris, France, in 1990, and the Diplôme d'Ingénieur and Ph.D. degrees from Ecole des Mines de Paris, Paris, France, in 1992 and 1996, respectively.

He spent one year as a Postdoctoral Fellow in the Control and Dynamical Systems Department at the California Institute of Technology, Pasadena. Since 1997, he has been Chargé de Recherche at the Institut National de Recherche en Automatique et Informatique (INRIA), Sophia, France. His research interests include control of nonlinear systems and its applications to mechanical systems.

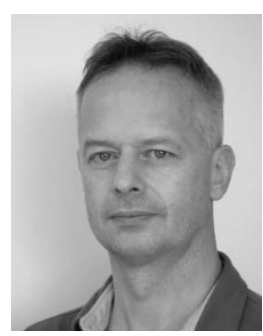

Claude Samson was born in Buenos Aires, Argentina, in 1955. After graduating from the Ecole Supérieure d'Electricité, Paris, France, in 1977, he received the Docteur-Ingénieur and Docteur d'Etat degrees from the University of Rennes, Rennes, Francem in 1980 and 1983, respectively.

$\mathrm{He}$ joined the Institut National de Recherche en Automatique et Informatique (INRIA) in 1981, where he is currently Directeur de Recherche and the head of the research group Icare whose activities are centered on robotics and related control issues. His research interests are in control theory and its applications to the control of mechanical systems. He is the coauthor, with M. Leborgne and B. Espiau, of the book Robot Control. The Task-Function Approach (Oxford, U.K.: Oxford University Press, 1991). 